\title{
A time-splitting pseudospectral method for the solution of the Gross-Pitaevskii equations using spherical harmonics with generalised-Laguerre basis functions
}

\author{
Hayder Salman \\ School of Mathematics, University of East Anglia, Norwich Research Park, Norwich, NR4 7TJ, United Kingdom
}

\section{A R T I C L E I N F O}

\section{Article history:}

Received 1 January 2013

Received in revised form 9 September 2013

Accepted 6 October 2013

Available online 22 October 2013

\section{Keywords:}

Gross-Pitaevskii equation

Generalised-Laguerre basis functions

Spherical harmonics

Bose-Einstein condensates

Nonlinear Schrödinger equation

Strang operator splitting

\begin{abstract}
A B S T R A C T
We present a method for numerically solving a Gross-Pitaevskii system of equations with a harmonic and a toroidal external potential that governs the dynamics of one- and two-component Bose-Einstein condensates. The method we develop maintains spectral accuracy by employing Fourier or spherical harmonics in the angular coordinates combined with generalised-Laguerre basis functions in the radial direction. Using an error analysis, we show that the method presented leads to more accurate results than one based on a sine transform in the radial direction when combined with a time-splitting method for integrating the equations forward in time. In contrast to a number of previous studies, no assumptions of radial or cylindrical symmetry is assumed allowing the method to be applied to 2D and 3D time-dependent simulations. This is accomplished by developing an efficient algorithm that accurately performs the generalised-Laguerre transforms of rotating Bose-Einstein condensates for different orders of the Laguerre polynomials. Using this spatial discretisation together with a second order Strang time-splitting method, we illustrate the scheme on a number of $2 \mathrm{D}$ and $3 \mathrm{D}$ computations of the ground state of a non-rotating and rotating condensate. Comparisons between previously derived theoretical results for these ground state solutions and our numerical computations show excellent agreement for these benchmark problems. The method is further applied to simulate a number of time-dependent problems including the Kelvin-Helmholtz instability in a twocomponent rotating condensate and the motion of quantised vortices in a 3D condensate.
\end{abstract}

(c) 2013 Elsevier Inc. All rights reserved.

\section{Introduction}

Since the experimental realisation of Bose-Einstein condensates (BECs) in 1995 [3,19], much progress has been made in uncovering the fundamental dynamical properties of these systems. In particular, experiments are now routinely carried out to study ensembles of particles in which quantised vortices [34], collective excitations of the condensate [33,24], soliton dynamics [35], and superfluid flow past impurities [4], among many other problems. Comparisons between these experiments and predictions obtained with the model of Gross and Pitaevskii [41,29] that describes the condensate dynamics have served to validate the broad relevance and applicability of this model. The Gross-Pitaevskii equation (GPE) provides a mean-field description for the dynamics of the condensate in which the motion of an atom moving in the effective potential arising from the interactions with all the other atoms is described by a nonlinear term. The coefficient appearing in front of this nonlinear term can in general be either a positive or negative constant depending on whether the interactions are repulsive or attractive. In fact, nowadays, the strength of these interactions can be carefully tuned using the experimental technique of Feshbach resonance [40]. The resulting GPE is then of the form of a Nonlinear Schrödinger (NLS) equation of the defocussing/self-focussing type corresponding to repulsive/attractive interactions, respectively. Given the excellent agreement 
with experiments and the widespread applicability of the GPE, there is a clear need to develop more accurate and efficient numerical schemes for the solution of the GPE system of equations for single and multi-component condensates.

Since the GPE is effectively a NLS equation with an external trapping potential, many methods developed for the latter can be applied to the former (see e.g. [46,17,30,22,49,39]). In fact, since the form of the nonlinearity in the equation is relatively simple, being a cubic algebraic nonlinearity which is local in physical space, the main challenge arises in accurately discretising the linear operator of the GPE. However, in formulating our method, we focus on experimental configurations and corresponding numerical issues that stem from having to model BECs in a trapping potential. At the same time, given that the GPE system we consider are derivable from an underlying Hamiltonian, we will seek a numerical scheme that takes into account the underlying symplectic structure of these equations [21,37].

In most experiments, the gas is confined within a harmonic trap. The external potential is then typically of the form $V(\mathbf{x})=m\left(\omega_{x}^{2} x^{2}+\omega_{y}^{2} y^{2}+\omega_{z}^{2} z^{2}\right) / 2$ where $\omega_{x}, \omega_{y}$, and $\omega_{z}$ are the oscillator frequencies in the three spatial directions. When the oscillator frequencies are equal, one recovers a spherically symmetric trap. Otherwise, if two of the frequencies are equal, we recover either a cigar shaped condensate (if $\omega_{x} \ll \omega_{y}$ and $\omega_{y} \sim \omega_{z}$ ), or a pancake shaped trap (if $\omega_{x} \sim \omega_{y}$ and $\left.\omega_{y} \ll \omega_{z}\right)$. In the latter case, if $\omega_{z}$ is sufficiently large relative to the thermal wavelength $\sqrt{h^{2} /\left(2 \pi m k_{B} T\right)}$, where $h$ is Planck's constant, $m$ is the atomic mass of the trapped gas, $k_{B}$ is Boltzmann's constant and $T$ is temperature, then the dynamics in this direction are frozen out and we can model the condensate as a 2D system. In all these cases, the circular or spherical geometry that is inherent in many experimental configurations motivates the use of a numerical discretisation that exploits this symmetry. There are a number of reasons for this. By exploiting the inherent symmetry through the use of spherical or polar coordinates in computations, a more optimal distribution of grid points can be used in simulations. When using a spectral/pseudospectral method, this corresponds to a more uniform truncation in spectral space. Moreover, for 3D problems, this eliminates the need to wastefully distribute points at the eight corners of the cube if a Cartesian mesh is used. When interpreted in terms of a Fourier basis, the use of a Cartesian mesh corresponds to introducing a nonuniform cut-off in wavenumber (or equivalently momentum) space. In contrast to other attempts, in this work we will combine the use of Fourier or Spherical harmonics for polar or spherical coordinates in 2D and 3D respectively, with generalised-Laguerre basis functions in the radial direction. As we will show in this paper, this basis has certain more desirable properties that lead to improved accuracy over alternative methods that are often used to discretise the radial coordinate.

One of the primary motivations for using the generalised-Laguerre basis is that it provides careful control of the energy cut-off which turns out to be particularly important when the GPE is extended to model BECs at finite temperature. As pointed out by a number of authors, who use extensions and generalisations of the GPE to model finite temperature effects $[15,16,43,20]$, a uniform cut-off in the energy space is essential in order to correctly model the system under these conditions. In such cases, all the spectral modes of the GP equation are macroscopically occupied and a judicious choice of the cut-off in the energy spectrum is essential for the successful application of such methods in modelling finite temperature effects. It turns out that these kind of considerations that arise for finite temperature systems lead one to seek the most consistent numerical schemes for the solution of the GPE. Therefore, while we will not specifically address finite temperature simulations in this work, we will proceed motivated by the realisation that numerical methods that impose a uniform cut-off on the spectral truncation (to be defined more precisely later) result in very efficient and accurate numerical approximations. By using Laguerre polynomials, we can develop methods with spectral-like accuracy. At the same time, the generalised-Laguerre polynomials have the desirable property that they correspond to the eigenmodes of the linear operator of the GPE in polar/spherical coordinates with a harmonic trap. Given these observations, the generalised-Laguerre polynomials appear to be the method of choice for our problem. Therefore, while a number of methods have already been developed in several different contexts for solving the linear Schrödinger equation in polar and spherical coordinates [30,22], they do not meet our criteria. For example, the work of [30] employs a sine transform which requires an artificial truncation of the physical domain. Sine transforms were also used by Bao and Jaksch [5] to solve the GPE on a finite domain using a time-splitting algorithm. The artificial truncation of the domain had to be performed at sufficiently large $r$ for the Dirichlet boundary condition $\psi=0$ that is imposed by a sine transform to be valid, hence leading to wasteful use of grid points. The main motivation for using Dirichlet boundary conditions of this type, which has also been adopted by [46], is that it permits the use of more familiar basis functions with well-understood properties to be applied to the problem. However, as shown by Boyd et al. [18], such a scheme tends to be less desirable than one based on Laguerre polynomials even when solving the eigenvalue problem. In addition, we will show in this paper that, when this spatial discretisation is used along with a time-splitting algorithm, significant time-splitting errors can arise. The desire to retain a time-splitting integration scheme follows from the excellent numerical properties that these methods have particularly for systems with an underlying Hamiltonian structure and in which the Hamiltonian is given by a kinetic energy plus a potential energy contribution [36,37]. As we show in Section 3, the problem with the splitting error that originates from the singular terms in the Laplacian that we identify in this paper, are also remedied by the use of a generalised-Laguerre basis for simulating the dynamics of Bose-Einstein condensates.

For a long time, the use of Laguerre polynomials for pseudospectral simulations had not received the widespread popularity enjoyed by other basis functions such as Legendre or Chebyshev spectral approximations [26]. Amongst the first attempts to use Laguerre polynomials for solving partial differential equations was the work of Gottlieb and Orszag [28] However, there has been a revived interest in their use. In the context of the GPE system, a series of papers have appeared in recent years describing a range of different pseudospectral schemes based on Hermite and Laguerre basis functions. In particular, the use of Laguerre basis functions for the GPE has been presented by Shen, and Bao and Shen [45,6]. Some 
of their earlier work assumed cylindrical or spherical symmetry. More recently, this assumption has been relaxed in [7]. The method was later reformulated in terms of a time-splitting scheme in [8] to retain the symplectic structure under the temporal evolution. A thorough review of the key methods formulated for modelling Bose-Einstein condensates can also be found in [9]. As correctly pointed out by these authors, Laguerre polynomials automatically satisfy the decaying boundary conditions of the wavefunction at infinity. Their use, therefore, circumvents the need to artificially truncate the domain at some large value of $r$ and then impose a Dirichlet boundary condition that sets the wavefunction to zero. This is because the eigenvalues of the Laguerre polynomials correspond to the energies of different modes. Therefore, provided a sufficiently large number of modes is retained in the computation, which depends on the initial total energy of the system, the particles will remain confined within the trap. This is in contrast to the problem of applying non-reflecting boundary conditions in the absence of a trapping potential which is a much more challenging issue as discussed in [32]. While the method we develop bears many similarities with the approach presented in [8], we emphasise the key differences between our formulation and theirs. In particular,

(i) We develop our 3D scheme using spherical harmonics which is the most natural coordinate system to use under certain experimental conditions, such as when the harmonic trapping frequencies are equal. This also produces a more uniform energy cut-off than the method of Bao et al. [8] who employed a cylindrical coordinate system in 3D in which generalised-Laguerre polynomials are combined with Hermite polynomials to represent the wavefunction.

(ii) As we explain later in the paper, in the scheme presented by Bao, a nonuniform truncation in energy is used for modes with different angular wavenumbers resulting in an inconsistent evolution of the modes that can be described by the discretised wavefunctions which are evaluated at a set of predefined collocation points. In contrast, we retain modes based on a consistent energy cut-off for all angular modes within each component.

(iii) We extend the application of our method to toroidal traps which again are naturally described by a spherical coordinate system.

(iv) One of the main challenges when using generalised-Laguerre polynomials occurs when the optimal choice of the collocation points for the exact evaluation of numerical quadratures turn out to be different for different components of a multi-component Bose gas. This typically occurs when the mass ratios of the different atomic species corresponding to each component become different. This is a very important problem since, as illustrated by a number of relevant studies considered in this work, important new effects can arise in such scenarios which are certainly within the capabilities of current experiments. We, therefore, formulate our pseudospectral method for a two-component system in a way that allows us to model these additional scenarios.

(v) Efficient implementation of the method by formulating the entire scheme in terms of matrix-matrix or matrix-vector operations that can be readily carried out using standard libraries and/or numerical software packages.

The paper is organised as follows. In part 2, we present the governing Gross-Pitaevskii model it in terms of polar and spherical coordinates that are relevant for the development of our numerical scheme for 2D and 3D simulations respectively. In Section 3, we present the second order accurate time-splitting algorithm of Strang [47]. The key difficulties that stem from using a time-splitting algorithm in radial or spherical coordinates are pointed out and illustrated through an error analysis. This is used to motivate the use of generalised-Laguerre polynomials. In Section 4, we present our spatial discretisation where we introduce generalised-Laguerre polynomials as our basis functions. A key aspect of the discussion is the efficient implementation of the numerical algorithm. The method is then applied to a number of model problems in Section 5 followed by conclusions in Section 6.

\section{The governing system of equations}

We are interested in modelling the dynamics of weakly interacting one- or two-component Bose-condensed gases at zero temperature $(T=0)$ in the presence of a confining external potential which are governed by one or two coupled Gross-Pitaevskii (GP) [40] equations, respectively. In this work, we will consider the dynamics of both an effective 2D and a 3D condensate. After non-dimensionalising our equations as shown in Appendix A, we express our equations in polar (2D) and spherical (3D) coordinates. For polar coordinates, where

$$
x=r \cos \theta, \quad y=r \sin \theta,
$$

and $r \in[0, \infty), \theta \in[0,2 \pi)$, our equations reduce to

$$
\begin{aligned}
i \frac{\partial \psi_{1}}{\partial t} & =\left[\frac{-1}{2} \frac{\partial^{2}}{\partial r^{2}}-\frac{1}{2 r} \frac{\partial}{\partial r}-\frac{1}{2 r^{2}} \frac{\partial^{2}}{\partial \theta^{2}}+V_{1}(\mathbf{r})+\gamma_{11}^{(2)}\left|\psi_{1}\right|^{2}+\gamma_{12}^{(2)}\left|\psi_{2}\right|^{2}+i \Omega_{z} \frac{\partial}{\partial \theta}\right] \psi_{1}, \\
i \frac{\partial \psi_{2}}{\partial t} & =\left[\frac{-\delta}{2} \frac{\partial^{2}}{\partial r^{2}}-\frac{\delta}{2 r} \frac{\partial}{\partial r}-\frac{\delta}{2 r^{2}} \frac{\partial^{2}}{\partial \theta^{2}}+\frac{1}{\delta} V_{2}(\mathbf{r})+\gamma_{22}^{(2)}\left|\psi_{2}\right|^{2}+\gamma_{21}^{(2)}\left|\psi_{1}\right|^{2}+i \Omega_{z} \frac{\partial}{\partial \theta}\right] \psi_{2} .
\end{aligned}
$$

The external trapping potential is given by 


$$
\begin{aligned}
V_{\alpha}(\mathbf{r}) & =\frac{r^{2}}{2}+V_{\alpha, \text { res }}(\mathbf{r})+V_{\alpha, \operatorname{tr}}(\mathbf{r}) \\
& =\frac{r^{2}}{2}+\frac{\left(\lambda_{x, \alpha}^{2}-1\right) x^{2}+\left(\lambda_{y, \alpha}^{2}-1\right) y^{2}}{2}+\lambda_{\mathrm{tr}, \alpha}^{2} \exp \left(\frac{-2 r^{2}}{l_{\mathrm{tr}, \alpha}^{2}}\right)
\end{aligned}
$$

where $V_{\text {res }}(\mathbf{r})$ is the residual part of the harmonic potential arising from the anisotropic contributions. For spherical coordinates in $3 \mathrm{D}$ where we have

$$
x=r \cos \theta \sin \phi, \quad y=r \sin \theta \sin \phi, \quad z=r \cos \phi,
$$

and $r \in[0, \infty), \theta \in[0,2 \pi), \phi \in[0, \pi]$, our equations transform to

$$
\begin{aligned}
i \frac{\partial \psi_{1}}{\partial t}= & {\left[\frac{-1}{2} \frac{\partial^{2}}{\partial r^{2}}-\frac{1}{r} \frac{\partial}{\partial r}-\frac{1}{2 r^{2} \sin ^{2} \phi}\left\{\frac{\partial^{2}}{\partial \theta^{2}}+\sin \phi \frac{\partial}{\partial \phi}\left(\sin \phi \frac{\partial}{\partial \phi}\right)\right\}\right.} \\
& \left.+V_{1}(\mathbf{r})+\gamma_{11}^{(3)}\left|\psi_{1}\right|^{2}+\gamma_{12}^{(3)}\left|\psi_{2}\right|^{2}+i \Omega_{z} \frac{\partial}{\partial \theta}\right] \psi_{1}, \\
\frac{\partial \psi_{2}}{\partial t}= & {\left[\frac{-\delta}{2} \frac{\partial^{2}}{\partial r^{2}}-\frac{\delta}{r} \frac{\partial}{\partial r}-\frac{\delta}{2 r^{2} \sin ^{2} \phi}\left\{\frac{\partial^{2}}{\partial \theta^{2}}+\sin \phi \frac{\partial}{\partial \phi}\left(\sin \phi \frac{\partial}{\partial \phi}\right)\right\}\right.} \\
& \left.+\frac{1}{\delta} V_{2}(\mathbf{r})+\gamma_{22}^{(3)}\left|\psi_{2}\right|^{2}+\gamma_{21}^{(3)}\left|\psi_{1}\right|^{2}+i \Omega_{z} \frac{\partial}{\partial \theta}\right] \psi_{2},
\end{aligned}
$$

and now

$$
\begin{aligned}
V_{\alpha}(\mathbf{r}) & =\frac{r^{2}}{2}+V_{\alpha, \text { res }}(\mathbf{r})+V_{\alpha, \operatorname{tr}}(\mathbf{r}) \\
& =\frac{r^{2}}{2}+\frac{\left(\lambda_{x, \alpha}^{2}-1\right) x^{2}+\left(\lambda_{y, \alpha}^{2}-1\right) y^{2}+\left(\lambda_{z, \alpha}^{2}-1\right) z^{2}}{2}+\lambda_{\mathrm{tr}, \alpha}^{2} \exp \left(\frac{-2 r^{2} \sin ^{2} \phi}{l_{\mathrm{tr}, \alpha}^{2}}\right) .
\end{aligned}
$$

To recast the Laplacian in the radial coordinate into a form that is similar to its Cartesian representation and hence eliminate the first derivative in $r$, it is common to introduce the reduced wavefunction $\Phi=r^{(d-1) / 2} \psi$. The above equations for the first component then reduces to

$$
\begin{aligned}
i \frac{\partial \Phi_{1}}{\partial t} & =\left[\frac{-1}{2} \frac{\partial^{2}}{\partial r^{2}}+\frac{\hat{\Lambda}^{2}(d)}{2 r^{2}}+V_{1}(\mathbf{r})+\gamma_{11}\left|\psi_{1}\right|^{2}+\gamma_{12}\left|\psi_{2}\right|^{2}+i \Omega_{z} \frac{\partial}{\partial \theta}\right] \Phi_{1}, \\
i \frac{\partial \Phi_{2}}{\partial t} & =\left[\frac{-\delta}{2} \frac{\partial^{2}}{\partial r^{2}}+\frac{\delta \hat{\Lambda}^{2}(d)}{2 r^{2}}+\frac{1}{\delta} V_{2}(\mathbf{r})+\gamma_{21}\left|\psi_{1}\right|^{2}+\gamma_{22}\left|\psi_{2}\right|^{2}+i \Omega_{z} \frac{\partial}{\partial \theta}\right] \Phi_{2},
\end{aligned}
$$

where $\delta=m_{1} / m_{2}>1$ is the ratio of the masses of the atomic species. For brevity, we have introduced the angular momentum operator $\hat{\Lambda}^{2}(d)$ which is defined as

$$
\begin{aligned}
& \hat{\Lambda}^{2}(2) \equiv\left\{-\frac{1}{4}-\frac{\partial^{2}}{\partial \theta^{2}}\right\}, \\
& \hat{\Lambda}^{2}(3) \equiv\left\{-\frac{1}{\sin ^{2} \phi}\left[\frac{\partial^{2}}{\partial \theta^{2}}+\sin \phi \frac{\partial}{\partial \phi}\left(\sin \phi \frac{\partial}{\partial \phi}\right)\right]\right\},
\end{aligned}
$$

in $2 \mathrm{D}$ and $3 \mathrm{D}$ respectively.

\section{Time integration}

Given the underlying Hamiltonian structure of our system, we will use a symplectic time integration scheme. A method that has become very popular for the GP equation $[27,50,36,38,46]$, in part due to its relative simplicity and desirable numerical properties, is the second order Strang time-splitting algorithm [47]. While higher order extensions of the time-splitting scheme have been developed and used for the GP equation (e.g. Bao et al. [6] used a 4th order time-splitting scheme), in this work it will suffice to consider the second order method such as that used in [46]. To motivate our approach, we will consider two different forms of the time-splitting scheme. We begin by rewriting our coupled GP equations for the reduced wavefunctions in the form

$$
i \frac{\partial \Phi_{\alpha}}{\partial t}=\left(\hat{\mathcal{L}}_{\alpha}+\hat{\mathcal{N}}_{\alpha}\right) \Phi_{\alpha},
$$

where $\hat{\mathcal{L}}_{\alpha}$ and $\hat{\mathcal{N}}_{\alpha}$ correspond to the linear and nonlinear operators given by 


$$
\begin{aligned}
& \hat{\mathcal{L}}_{\alpha}=\hat{\mathcal{L}}_{\alpha, \text { rad }}+\hat{\mathcal{L}}_{\alpha, \text { ang }}, \quad \text { where } \hat{\mathcal{L}}_{\alpha, \text { rad }} \equiv-\frac{\delta_{\alpha}}{2} \frac{\partial^{2}}{\partial r^{2}}, \hat{\mathcal{L}}_{\alpha, \text { ang }} \equiv \frac{\delta_{\alpha} \hat{\Lambda}^{2}(d)}{2 r^{2}}+\frac{r^{2}}{2 \delta_{\alpha}}+i \Omega_{z} \frac{\partial}{\partial \theta}, \\
& \hat{\mathcal{N}}_{1}=\gamma_{11}\left|\psi_{1}\right|^{2}+\gamma_{12}\left|\psi_{2}\right|^{2}+V_{1, \text { res }}(\mathbf{r})+V_{1, \text { tr }}(\mathbf{r}), \\
& \hat{\mathcal{N}}_{2}=\gamma_{21}\left|\psi_{1}\right|^{2}+\gamma_{22}\left|\psi_{2}\right|^{2}+\frac{1}{\delta} V_{2, \text { res }}(\mathbf{r})+\frac{1}{\delta} V_{2, \operatorname{tr}}(\mathbf{r}) .
\end{aligned}
$$

Having further split the linear operator into a radial and an angular part, Eq. (10) can then be integrated forward in time using a Strang method with a three operator-splitting $[37,46]$ so that

$$
\Phi_{\alpha}\left(t_{n+1}\right)=\mathrm{e}^{-i \frac{\Delta t}{2} \hat{\mathcal{N}}_{\alpha}} \mathrm{e}^{\frac{-i \Delta t}{2}} \hat{\mathcal{L}}_{\alpha, \text { ang }} \mathrm{e}^{-i \Delta t \hat{\mathcal{L}}_{\alpha, \text { rad }}} \mathrm{e}^{\frac{-i \Delta t}{2}} \hat{\mathcal{L}}_{\alpha, \text { ang }} \mathrm{e}^{-i \frac{\Delta t}{2} \hat{\mathcal{N}}_{\alpha}} \Phi_{\alpha}\left(t_{n}\right)+\mathcal{O}\left(\Delta t^{3}\right)
$$

for a given time step $\Delta t$ where $t_{n}=n \Delta t$, with $n=0,1,2, \ldots$, and $\Phi_{\alpha}^{n} \equiv \Phi_{\alpha}\left(\mathbf{x}, t_{n}\right)$. The splitting given above has a local error that is third order and a global error that is second order accurate in time [37]. The local error differential operator is given by

$$
\begin{aligned}
& \hat{E}(\mathbf{r}, \Delta t) \equiv\left(\mathrm{e}^{-i \Delta t\left(\hat{\mathcal{L}}_{\alpha}+\hat{\mathcal{N}}_{\alpha}\right)}-\mathrm{e}^{-i \frac{\Delta t}{2}} \hat{\mathcal{N}}_{\alpha} \mathrm{e}^{-\frac{i \Delta t}{2}} \hat{\mathcal{L}}_{\alpha, \text { ang }} \mathrm{e}^{-i \Delta t \hat{\mathcal{L}}_{\alpha, \text { rad }}} \mathrm{e}^{\frac{-i \Delta t}{2}} \hat{\mathcal{L}}_{\alpha, \text { ang }} \mathrm{e}^{-i \frac{\Delta t}{2}} \hat{\mathcal{N}}_{\alpha}\right) \\
& =\left(\frac{1}{24}\left[\hat{\mathcal{N}}_{\alpha},\left[\hat{\mathcal{N}}_{\alpha}, \hat{\mathcal{L}}_{\alpha, \text { rad }}\right]\right]-\frac{1}{12}\left[\hat{\mathcal{L}}_{\alpha, \text { rad }},\left[\hat{\mathcal{L}}_{\alpha, \text { rad }}, \hat{\mathcal{N}}_{\alpha}\right]\right) \Delta t^{3}\right. \\
& +\left(\frac{1}{24}\left[\hat{\mathcal{N}}_{\alpha}+\hat{\mathcal{L}}_{\alpha, \text { rad }},\left[\hat{\mathcal{N}}_{\alpha}+\hat{\mathcal{L}}_{\alpha, \text { rad }}, \hat{\mathcal{L}}_{\alpha, \text { ang }}\right]\right]-\frac{1}{12}\left[\hat{\mathcal{L}}_{\alpha, \text { ang }},\left[\hat{\mathcal{L}}_{\alpha, \text { ang }}, \hat{\mathcal{N}}_{\alpha}+\hat{\mathcal{L}}_{\alpha, \text { rad }}\right]\right) \Delta t^{3}\right. \\
& +\left(\frac{1}{8}\left[\hat{\mathcal{L}}_{\alpha, \text { ang }},\left[\hat{\mathcal{N}}_{\alpha}, \hat{\mathcal{L}}_{\alpha, \text { rad }}\right]\right) \Delta t^{3}+\mathcal{O}\left(\Delta t^{4}\right) .\right.
\end{aligned}
$$

From above, we see that the time evolution during one time step involves five update steps which we write explicitly as

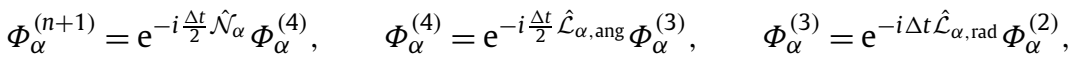

$$
\begin{aligned}
& \Phi_{\alpha}^{(2)}=\mathrm{e}^{-i \frac{\Delta t}{2} \hat{\mathcal{L}}_{\alpha, \text { ang }}} \Phi_{\alpha}^{(1)}, \quad \Phi_{\alpha}^{(1)}=\mathrm{e}^{-i \frac{\Delta t}{2} \hat{\mathcal{N}}_{\alpha}} \Phi_{\alpha}^{(n)} .
\end{aligned}
$$

We note that the steps involving the nonlinear operators are easily solved in physical space. Therefore, during the propagation by the nonlinear operator, $\hat{\mathcal{N}}_{1}$ say, $\Phi_{1}$ satisfies

$$
\begin{aligned}
\frac{d\left|\Phi_{1}\right|^{2}}{d t} & =\Phi_{1}^{*} \frac{d \Phi_{1}}{d t}+\Phi_{1} \frac{d \Phi_{1}^{*}}{d t} \\
& =-i \Phi_{1}^{*}\left[\gamma_{11}^{(d)}\left|\psi_{1}\right|^{2}+\gamma_{12}^{(d)}\left|\psi_{2}\right|^{2}+\left(V_{1, \text { res }}+V_{1, \text { rr }}\right)\right] \Phi_{1}+i \Phi_{1}\left[\gamma_{11}^{(d)}\left|\psi_{1}\right|^{2}+\gamma_{12}^{(d)}\left|\psi_{2}\right|^{2}+\left(V_{1, \text { res }}+V_{1, \text { tr }}\right)\right] \Phi_{1}^{*} \\
& =0 .
\end{aligned}
$$

A similar result holds for $\Phi_{2}$. It follows that an exact solution for the reduced wavefunctions $\Phi_{\alpha}$ is given by

$$
\Phi_{1}^{(1)}=\mathrm{e}^{-i \Delta t\left[\gamma_{11}\left|\psi_{1}\right|^{2}+\gamma_{12}\left|\psi_{2}\right|^{2}+\left(V_{1, \text { res }}+V_{1, \text { tr }}\right)\right] / 2} \Phi_{1}^{n} .
$$

It remains to find an accurate and efficient method to evolve the equations under the linear operator.

The motivation of the three way splitting, as used by other authors, now becomes clear since the nonlinear term and external potential can be easily calculated in physical space whereas the angular contribution from the Laplacian operator can be computed accurately using a Fourier transform in 2D, or using a spherical harmonic transform in 3D. To see this, we expand our reduced wavefunctions in the form

$$
\Phi_{\alpha}(r, \theta)=\sum_{m} R_{\alpha, m}(r) \mathrm{e}^{i m \theta} \quad \text { or } \quad \Phi_{\alpha}(r, \theta, \phi)=\sum_{l, m} R_{\alpha, l m}(r) Y_{l}^{m}(\theta, \phi),
$$

for 2D and 3D, respectively. The orthonormal spherical harmonics are defined as

$$
Y_{l}^{m}(\theta, \phi)=\sqrt{\frac{(2 l+1)}{4 \pi} \frac{(l-m) !}{(l+m) !}} P_{l}^{m}(\cos \phi) \mathrm{e}^{i m \theta}, \quad l=0,1,2, \ldots, m=-l,-l+1, \ldots, l-1, l
$$

where $P_{l}^{m}(x)$ are the associated Legendre polynomials [1].

The advantage of working with such a basis is that the angular momentum operators simplify drastically in either case leading to

$$
\begin{aligned}
& \hat{\Lambda}^{2}(2) \Phi_{\alpha}(r, \theta)=\sum_{m} R_{\alpha, m}(r)\left(m^{2}-\frac{1}{4}\right) \mathrm{e}^{i m \theta}, \quad m=0, \pm 1, \pm 2, \ldots, \\
& \hat{\Lambda}^{2}(3) \Phi_{\alpha}(r, \theta, \phi)=\sum_{m, l} R_{\alpha, l m}(r) l(l+1) Y_{l}^{m}(\theta, \phi), \quad l=0,1,2, \ldots, m=-l,-l+1, \ldots, l-1, l .
\end{aligned}
$$


Therefore, after evolving the reduced wavefunctions $\Phi_{\alpha}^{n}$ under the nonlinear operator, that includes contributions from the parts of the external potential corresponding to $V_{\alpha, \text { tr }}$ and $V_{\alpha, \text { res }}$, we obtain $\Phi_{\alpha}^{(1)}$. A Fourier transform or Spherical harmonic transform is then performed leading to the transformed reduced wavefunction $\tilde{\Phi}_{\alpha}^{(1)}$ which depends on the angular wavenumbers $m$ in 2D, and $l$ and $m$ in 3D. The governing equation under the angular momentum operators in this case can be solved so that

$$
\tilde{\Phi}_{\alpha, m}^{(2)}=\mathrm{e}^{-i \Delta t\left[\frac{\left(m^{2}-1 / 4\right)}{4 r^{2}}+\frac{r^{2}}{4}\right]} \tilde{\Phi}_{\alpha, m}^{(1)}, \quad \text { in 2D, } \quad \tilde{\Phi}_{\alpha, l m}^{(2)}=\mathrm{e}^{-i \Delta t\left[\frac{l(l+1)}{4 r^{2}}+\frac{r^{2}}{4}\right]} \tilde{\Phi}_{\alpha, l m}^{(1)}, \quad \text { in 3D. }
$$

The evolution under the angular momentum operator is therefore computed exactly under such a transformation. Time integration of the wavefunction under the radial Laplacian operator can then be carried out for a suitably chosen basis. For example, a sine transform could be used.

At first sight, the 3-way splitting presented above appears to result in a highly accurate symplectic integration scheme with the only caveat that the far-field boundary conditions are approximated by an artificial truncation of the computational domain. However, upon more careful inspection of the splitting error given by Eq. (13), we note that a severe degrading of the accuracy of the scheme results from the singular terms in the angular momentum operators. In particular, if we focus on the splitting error originating purely from the Laplacian term, then we see that even for the linear Schrödinger equation with a cylindrically (2D) or spherically (3D) symmetric harmonic trap (i.e. where $\hat{\mathcal{N}}=0$ ), the error given by Eq. (13) reduces to

$$
\hat{E}(\mathbf{r}, \Delta t)=\left(\frac{1}{24}\left[\hat{\mathcal{L}}_{\alpha, \text { rad }},\left[\hat{\mathcal{L}}_{\alpha, \text { rad }}, \hat{\mathcal{L}}_{\alpha, \text { ang }}\right]\right]-\frac{1}{12}\left[\hat{\mathcal{L}}_{\alpha, \text { ang }},\left[\hat{\mathcal{L}}_{\alpha, \text { ang }}, \hat{\mathcal{L}}_{\alpha, \text { rad }}\right]\right) \Delta t^{3} .\right.
$$

Focusing on the case without rotation (i.e. $\Omega_{z}=0$ ), we obtain

$$
\hat{E}(\mathbf{r}, \Delta t)=\frac{\Delta t^{3} \delta_{\alpha}^{3} \hat{\Lambda}^{2}(d)}{192}\left(\frac{120}{r^{4}}-\frac{12}{r^{4}} \frac{\partial^{2}}{\partial r^{2}}+\frac{4}{r^{3}} \frac{\partial^{3}}{\partial r^{3}}\right)+\frac{\Delta t^{3} \delta_{\alpha}^{3} \hat{\Lambda}^{4}(d)}{96}\left(\frac{1}{r^{4}} \frac{\partial^{2}}{\partial r^{2}}-\frac{6}{r^{8}}\right)+\hat{E}_{\mathrm{reg}}(\mathbf{r}, \Delta t),
$$

where $\hat{E}_{\text {reg }}(\mathbf{r}, \Delta t)$ denotes the contribution to the error arising from the regular $r^{2} / 2$ term contained in the linear operator $\hat{\mathcal{L}}_{\alpha, \text { ang. }}$. A striking conclusion from this is that the error is most severe near the origin. In addition, while this error arises only when the spherical symmetry is lost in 3D (i.e. for $l \neq 0$ ), it is present even in the radially symmetric case in $2 \mathrm{D}$ due to the $(-1 / 4)$ constant term appearing in the definition of $\hat{\Lambda}^{2}(2)$ (see Eq. (8)). Since the error increases near the origin and is associated with the operator splitting, using a high order discretisation in space and a fine grid cannot resolve this problem. In fact, as we refine the computational grid, the error worsens since our collocation points are located more closely to the origin resulting in a larger contribution to the truncation error from the singular terms in Eq. (22). Moreover, the error is worsened by seeking a higher order splitting. Indeed, when we compare with a first order splitting scheme given by

$$
\Phi_{\alpha}\left(t_{n+1}\right)=\mathrm{e}^{-i \Delta t \hat{\mathcal{L}}_{\alpha, \text { rad }}} \mathrm{e}^{-i \Delta t \hat{\mathcal{L}}_{\alpha, \text { ang }}} \Phi_{\alpha}\left(t_{n}\right)+\mathcal{O}\left(\Delta t^{2}\right),
$$

with an error given by

$$
\hat{E}(\mathbf{r}, \Delta t)=\left[\hat{L}_{\alpha, \text { rad }}, \hat{L}_{\alpha, \text { ang }}\right] \Delta t^{2}=\frac{\Delta t^{2} \delta_{\alpha}^{2} \hat{\Lambda}^{2}(d)}{4}\left(\frac{1}{r^{2}} \frac{\partial^{2}}{\partial r^{2}}-\frac{6}{r^{4}}\right),
$$

we see that while the error is worst at the origin, it is nevertheless less singular with respect to $r$ than the second order Strang splitting. Therefore, increasing the order of the scheme worsens the problem. This type of splitting error was also noted by Sørevik [46] who considered the linear Schrödinger equation with a Coulomb potential. This observation, together with our goal to develop a scheme that provides more accurate control of the spectral truncation of the modes, is further motivation for the use of generalised-Laguerre polynomials as basis functions. This basis resolves essentially all of the complications identified here that would otherwise arise by using sine basis functions for the radial coordinate. We will, therefore, proceed by reverting to a symmetric two operator splitting of the form given in Eq. (10) where no further splitting is performed on the linear operator $\hat{\mathcal{L}}_{\alpha}$.

This leads to a scheme similar to the one described in Bao et al. [8] in which the reduced wavefunctions are evolved forward in time using

$$
\Phi_{\alpha}^{n+1}=\mathrm{e}^{\frac{-i \Delta t}{2} \hat{\mathcal{N}}_{\alpha}} \mathrm{e}^{-i \Delta t \hat{\mathcal{L}}_{\alpha}} \mathrm{e}^{\frac{-i \Delta t}{2}} \hat{\mathcal{N}}_{\alpha} \Phi_{\alpha}^{n}+\mathcal{O}\left(\Delta t^{3}\right) .
$$

The splitting error in this case is given by

$$
\begin{aligned}
E(\mathbf{x}, \Delta t) & \equiv\left(\mathrm{e}^{-i \Delta t\left(\hat{\mathcal{L}}_{\alpha}+\hat{\mathcal{N}}_{\alpha}\right)}-\mathrm{e}^{\frac{-i \Delta t}{2}} \hat{\mathcal{N}}_{\alpha} \mathrm{e}^{-i \Delta t \hat{\mathcal{L}}_{\alpha}} \mathrm{e}^{\frac{-i \Delta t}{2} \hat{\mathcal{N}}_{\alpha}}\right) \\
& =\left(\frac{1}{24}\left[\hat{\mathcal{N}}_{\alpha},\left[\hat{\mathcal{N}}_{\alpha}, \hat{\mathcal{L}}_{\alpha}\right]\right]-\frac{1}{12}\left[\hat{\mathcal{L}}_{\alpha},\left[\hat{\mathcal{L}}_{\alpha}, \hat{\mathcal{N}}_{\alpha}\right]\right]\right) \Delta t^{3}+\mathcal{O}\left(\Delta t^{4}\right) .
\end{aligned}
$$

As a consistency check, it can be shown after some calculations that this is exactly what we would recover from Eq. (13), if we make the substitution $\mathcal{L}_{\alpha \text {, ang }} \rightarrow \frac{1}{2} \mathcal{L}_{\alpha}$, and $\mathcal{L}_{\alpha \text {,rad }} \rightarrow \frac{1}{2} \mathcal{L}_{\alpha}$ as expected. In the next section we describe how an accurate 
and numerically efficient implementation of a generalised-Laguerre transform can be implemented in both $2 \mathrm{D}$ and 3D to evolve the system under the full linear operator that we have defined above. The resulting scheme that we develop, therefore evolves the reduced wavefunctions in three steps according to

$$
\Phi_{\alpha}^{(1)}=\mathrm{e}^{-i \frac{\Delta t}{2} \hat{\mathcal{N}}_{\alpha}} \Phi_{\alpha}^{n}, \quad \Phi_{\alpha}^{(2)}=\mathrm{e}^{-i \Delta t \hat{\mathcal{L}}_{\alpha}} \Phi_{\alpha}^{(1)}, \quad \Phi_{\alpha}^{(n+1)}=\mathrm{e}^{-i \frac{\Delta t}{2} \hat{\mathcal{N}}_{\alpha}} \Phi_{\alpha}^{(2)} .
$$

In some of the results to be presented later in the paper, we will also be interested in computing the ground states of the condensates in various different configurations. The most straightforward way to compute these ground states is to evolve the system in imaginary time by introducing the transformation $\Delta t \rightarrow-i \Delta t$ in our time integration. This produces an evolution under a Ginzburg-Landau equation where the time evolution is no longer conservative but rather becomes dissipative. For these ground state computations, we no longer need to consider an accurate time evolution. Therefore, whenever computing ground states we have used a low-order Strang splitting given by

$$
\Phi_{\alpha}^{(1)}=\mathrm{e}^{-\Delta t\left(\hat{\mathcal{N}}_{\alpha}-\mu\right)} \Phi_{\alpha}^{n}, \quad \Phi_{\alpha}^{(n+1)}=\mathrm{e}^{-\Delta t \hat{\mathcal{L}}_{\alpha}} \Phi_{\alpha}^{(1)},
$$

where $\mu$ is the chemical potential. In general, the evolution given by Eq. (28) will neither conserve the number of particles $N_{\alpha}$, nor the total energy given by the Hamiltonian $H$. However, we need to compute the ground states subject to a given normalisation given by $N_{\alpha}=1$ in non-dimensional units. To achieve this, we rescale the modulus of the wavefunctions $\left|\psi_{\alpha}\right|$ at each time step to ensure that the normalisation condition is satisfied. In addition, at each time step, we evaluate the value of the chemical potential which is given by

$$
\begin{aligned}
\mu= & \left\{\int_{\mathbb{R}^{d}}\left[\sum_{\alpha=1}^{2}\left(\frac{\hbar^{2}}{2 m_{\alpha}}\left|\nabla \psi_{\alpha}(\mathbf{x}, t)\right|^{2}+V_{\alpha}(\mathbf{x})\left|\psi_{\alpha}(\mathbf{x}, t)\right|^{2}+U_{\alpha, \alpha}^{(d)}\left|\psi_{\alpha}(\mathbf{x}, t)\right|^{4}\right)+U_{12}^{(d)}\left|\psi_{1}(\mathbf{x}, t)\right|^{2}\left|\psi_{2}(\mathbf{x}, t)\right|^{2}\right] d^{d} \mathbf{x}\right. \\
& \left.-i \hbar \int_{\mathbb{R}^{d}}\left[\sum_{\alpha=1}^{2} \psi_{\alpha}^{*} \tilde{\boldsymbol{\Omega}} \cdot \mathbf{x} \times \nabla \psi_{\alpha}(\mathbf{x}, t)\right] d^{d} \mathbf{x}\right\} / \int\left|\psi_{\alpha}(\mathbf{x}, t)\right|^{2} d^{d} \mathbf{x} .
\end{aligned}
$$

This scheme guarantees that the system converges to a ground state with the prescribed normalisation as desired.

\section{Spatial discretisation}

\subsection{D Gross-Pitaevskii equations}

We begin by considering the 2D GP equations in polar coordinates. The results will be extended to the 3D case with spherical coordinates in the subsequent section. Having identified that a Fourier transform followed by a generalisedLaguerre transform is the appropriate way to proceed in evolving the linear operator as defined in the previous section, our starting point will be to address the solution of the linear operator appearing in the Strang time-splitting scheme given by Eq. (25). We will begin by providing a collocation method for performing the necessary transforms in an accurate and efficient manner. For the angular coordinate, we represent the wavefunction on a discrete set of collocation points $\theta_{j}=2 \pi j / M$ where, to fully utilise the power of the Fast Fourier Transform (FFT), $M$ is taken to be an integer power of 2 and $0 \leqslant j \leqslant M-1$. Furthermore, we discretise the wavefunction in the radial direction onto a set of, as yet, unspecified points $r_{\alpha, i}$ where $0 \leqslant i \leqslant K-1$. Note that we have retained the index $\alpha$ in anticipation of the need to use a different set of points for each component as we will discuss later. Hence, starting with Eq. (10), and focusing on the evolution of the reduced wavefunctions $\Phi_{\alpha}$ under the linear operator, we apply an FFT to transform the equation into the form

$$
i \frac{\partial R_{\alpha, m}\left(r_{\alpha, i}, t\right)}{\partial t}=\frac{-\delta_{\alpha}}{2} \frac{\partial^{2} R_{\alpha, m}\left(r_{\alpha, i}, t\right)}{\partial r^{2}}+\frac{\delta_{\alpha}\left(m^{2}-1 / 4\right) R_{\alpha, m}\left(r_{\alpha, i}, t\right)}{2 r_{\alpha, i}^{2}}+\frac{r_{\alpha, i}^{2}}{2 \delta_{\alpha}} R_{\alpha, m}\left(r_{\alpha, i}, t\right)-m \Omega_{z} R_{\alpha, m}\left(r_{\alpha, i}, t\right),
$$

which describes the evolution of the modes $R_{\alpha, m}$ at the set of points $\left\{r_{\alpha, i}\right\}$. This step can be performed very efficiently in $\mathcal{O}(M \log M)$ operations. Now following Baye and Heenen [12], we express $R_{\alpha, m}(r, t)$ as

$$
R_{\alpha, m}(r, t)=\sum_{i=0}^{K-1} R_{\alpha, m}\left(r_{\alpha, i}, t\right) f_{\alpha, i}(r)
$$

where $f_{\alpha, i}(r)$ are Lagrange functions satisfying the condition $f_{\alpha, i}\left(r_{\alpha, j}\right)=\delta_{i j}$. In other words, we seek a Lagrange mesh for the set of points $\left\{r_{\alpha, i}\right\}$ with a number of additional properties that fulfil the conditions to be specified in what follows. Firstly, our choice of the collocation points is made by requiring that the orthogonality condition

$$
\int_{0}^{\infty} f_{\alpha, i}^{*}(r) f_{\alpha, j}(r) d r=\lambda_{\alpha, i} \delta_{i j}
$$


is satisfied exactly for polynomials $f_{\alpha, i}^{*}(r) f_{\alpha, j}(r)$ of degree $2 K-1$ on the interval $[0, \infty)$. In order to satisfy the above orthogonality condition and determine our set of points $\left\{r_{\alpha, i}\right\}$, we consider a set of basis functions $\varphi_{\alpha, k}(r)$ that are orthonormal such that

$$
\int_{0}^{\infty} \varphi_{\alpha, k}^{*}(r) \varphi_{\alpha, k^{\prime}}(r) d r=\delta_{k, k^{\prime}}
$$

The existence of a Lagrange mesh for $f_{\alpha, i}(r)$ with points at $r_{\alpha, i}$ that satisfy the interpolating condition (31) together with the orthogonality condition (33) can then be obtained provided that

$$
f_{\alpha, i}\left(r_{\alpha, j}\right)=\lambda_{\alpha, i} \sum_{k=0}^{K-1} \varphi_{\alpha, k}^{*}\left(r_{\alpha, i}\right) \varphi_{\alpha, k}\left(r_{\alpha, j}\right)=\delta_{i j}, \quad \lambda_{\alpha, i}=\left(\sum_{k=0}^{K-1}\left|\varphi_{\alpha, k}\left(r_{\alpha, i}\right)\right|^{2}\right)^{-1}
$$

For our linear operator, a natural choice is provided by the polynomials

$$
\varphi_{\alpha, k}^{m}(r)=C_{\alpha, k m} r^{(|m|+1 / 2)} \mathrm{e}^{-r^{2} /\left(2 \delta_{\alpha}\right)} L_{k}^{|m|}\left(\frac{r^{2}}{\delta_{\alpha}}\right),
$$

which are expressed in terms of the generalised-Laguerre polynomials of degree $k$ and order $|m|$ and where the second index corresponds to the angular wavenumber. The normalisation condition is given by

$$
C_{\alpha, k m}=\left[\frac{2 k !}{\delta_{\alpha}^{m+1}(k+m) !}\right]^{1 / 2}
$$

The generalised-Laguerre polynomials are defined as

$$
L_{k}^{m}(r)=\frac{r^{-m} \mathrm{e}^{r}}{k !} \frac{d^{k}}{d r^{k}}\left(\mathrm{e}^{-r} r^{k+m}\right) .
$$

However, for numerical evaluation, we make use of the recurrence relation

$$
L_{0}^{m}(x)=1, \quad L_{1}^{m}(x)=1+m-x, \quad L_{n}^{m}(x)=\left(\frac{2 n+m-1-x}{n}\right) L_{n-1}^{m}(x)-\left(\frac{n+m-1}{n}\right) L_{n-2}^{m}(x) .
$$

They satisfy the orthogonality relation

$$
\int_{0}^{\infty} C_{\alpha, k|m|} C_{\alpha, k^{\prime}|m|} L_{k}^{|m|}\left(\frac{r^{2}}{\delta_{\alpha}}\right) L_{k^{\prime}}^{|m|}\left(\frac{r^{2}}{\delta_{\alpha}}\right) w(r) d r=\delta_{k, k^{\prime}}
$$

where the weight function is given by $w(r)=r^{(2|m|+1)} \mathrm{e}^{-r^{2} / \delta_{\alpha}}$. The key advantage of working with the Laguerre basis is that we can evaluate the linear operator exactly by making use of the corresponding eigenvalue equation for the generalisedLaguerre polynomials given by

$$
\left[-\frac{\delta_{\alpha}}{2} \frac{d^{2}}{d r^{2}}+\frac{\delta_{\alpha}\left(m^{2}-1 / 4\right)}{2 r^{2}}+\frac{r^{2}}{2 \delta_{\alpha}}-m \Omega\right] \varphi_{\alpha, k}^{m}=[(2 k+|m|+1)-m \Omega] \varphi_{\alpha, k}^{m}, \quad m=0, \pm 1, \pm 2, \ldots
$$

In terms of the generalised-Laguerre polynomials, the condition corresponding to Eq. (34) is given by

$$
\sum_{k=0}^{K_{m}-1} L_{k}^{|m|}\left(\frac{r_{\alpha, i}^{2}}{\delta_{\alpha}}\right) L_{k^{\prime}}^{|m|}\left(\frac{r_{\alpha, i}^{2}}{\delta_{\alpha}}\right) w\left(r_{\alpha, i}\right)=\lambda_{\alpha, i}^{-1} \delta_{k, k^{\prime}},
$$

where $K_{m}$ is an upper modal cut-off to be specified. In general, condition (39) can be computed exactly from $K$ collocation points for Laguerre polynomials of degree $K_{m}$ provided that $\left\{r_{\alpha, i}\right\}, 0 \leqslant i \leqslant K-1$, are chosen as the zeros of the orthonormal basis functions $\varphi_{\alpha, k}^{m}(r)$ for some $k \geqslant K_{m}$. However, in a typical simulation, our wavefunctions are spanned by Laguerre polynomials of different order $m$. Since the zeros of generalised-Laguerre polynomials of different order $m$ do not coincide, this leaves open the question of how to truncate the basis. In particular, we cannot simply truncate by using the same value of $K$ for Laguerre polynomials of different order since the effect of the order index $|\mathrm{m}|$ is to shift the zeros of the Laguerre polynomials further away from the origin. This is clearly illustrated in Fig. 1(a) in which two polynomials $\varphi_{1, k}^{m}$ of degree $k=16$ and order $m=0$ and $m=6$ are shown. As can clearly be seen from the figure, the zeros of the Laguerre polynomial of order 6 are shifted further away from the origin. Therefore, while using the zeros of the $\varphi_{1,16}^{0}$ polynomial is desirable in order to correctly represent our wavefunction near the origin, we would certainly misrepresent our wavefunction at larger radii if we simply retain an equal number of modes $K_{m}$ for Laguerre polynomials corresponding to a given angular wavenumber $m$ with such a set of collocation points. 


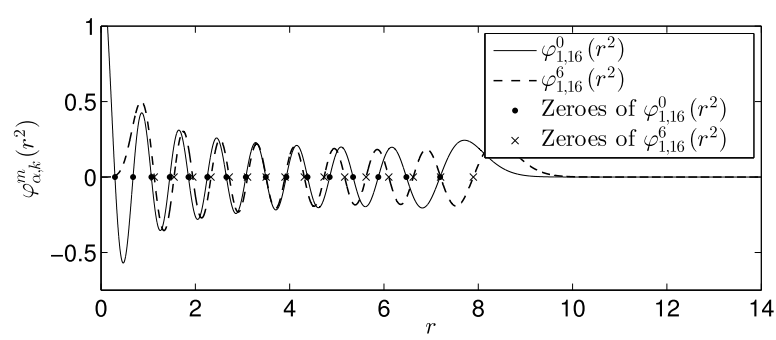

(a) Profiles and zeroes of polynomials for component $1\left(\delta_{1}=1\right)$ corresponding to $\varphi_{1,16}^{0}\left(r^{2}\right)$ and $\varphi_{1,16}^{6}\left(r^{2}\right)$.

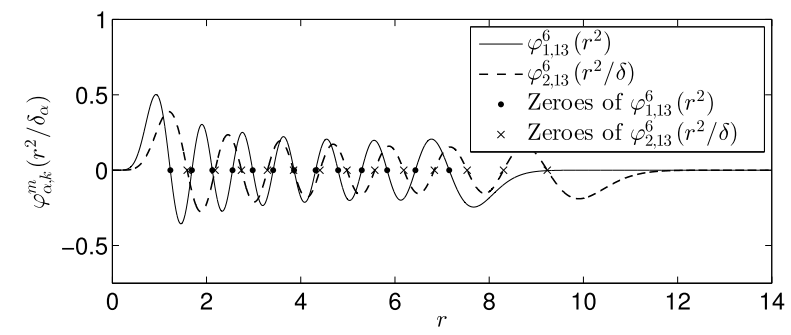

(c) Profiles and zeroes of polynomials for components $1\left(\delta_{1}=1\right)$ and $2\left(\delta_{2}=\delta=1.667\right)$ for $\varphi_{1,13}^{6}\left(r^{2}\right)$ and $\varphi_{2,13}^{6}\left(r^{2} / \delta\right)$.

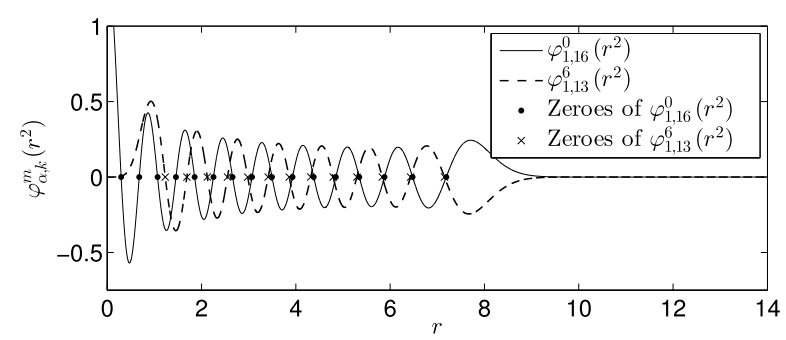

(b) Profiles and zeroes of polynomials for component $1\left(\delta_{1}=1\right)$ corresponding to $\varphi_{1,16}^{0}\left(r^{2}\right)$ and $\varphi_{1,13}^{6}\left(r^{2}\right)$.

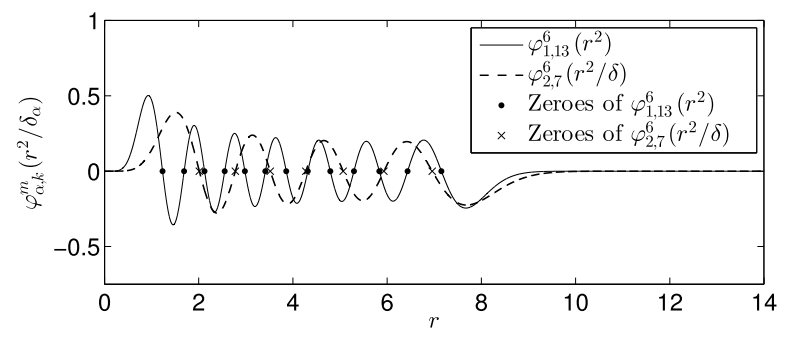

(d) Profiles and zeroes of polynomials for components $1\left(\delta_{1}=1\right)$ and $2\left(\delta_{2}=\delta=1.667\right)$ for $\varphi_{1,13}^{6}\left(r^{2}\right)$ and $\varphi_{2,7}^{6}\left(r^{2} / \delta\right)$.

Fig. 1. Comparison of different polynomials $\varphi_{\alpha, k}^{|m|}\left(r^{2} / \delta_{\alpha}\right)$ of degree $k$ and order $m$ for component $\alpha$ in 2D.

This leaves open the question of how to truncate the basis in a numerical scheme. For $\Omega=0$, it is clear that the energy (eigenvalue) corresponding to the $\varphi_{1,16}^{6}$ polynomial is larger than that corresponding to the $\varphi_{1,16}^{0}$ polynomial. To circumvent this difficulty, Bao et al. [8], employed the zeros of the Laguerre polynomial of degree $K+M / 2$ thereby ensuring that the orthogonality condition given by expression (41) remains exact for all modes in the system. At the same time, they retained a fixed number of modes, $K$, in the radial direction for each angular momentum wavenumber $|m|$. However, on physical grounds such a truncation would lead to a different energy cut-off for modes corresponding to different values of $|m|$. Moreover, the action of the residue external potential or the nonlinear term is to mix a given mode of the wavefunction into different modes. This means that modes corresponding to $|m|=0$ and with a degree $k>K$ can become populated. However these modes are not subsequently evolved under the linear operator leading to an inconsistent treatment of these modes relative to the modes lying within the spectral interval $|m|>0$ and $k \leqslant K+|m|$.

We note that the prefactor $r^{(|m|+1 / 2)}$ appearing in the definition of Eq. (35) contributes to the overall degree of the polynomials used as our basis. From this, and the expression for the energy given by the right-hand side of Eq. (40), it is clear that the cut-off wavenumber $K_{m}$ must be different for different values of the angular momentum number $m$. Since a consistent truncation of the energy for all modes is sound on physical grounds, we will proceed by choosing the zeros of the Laguerre polynomial of degree $K$ and order $m=0$ as the collocation points of our scheme. A truncation for the modes based on their energy, or equivalently the eigenvalues of the linear operator (with $\Omega=0$ ) would then ensure that the numerically computed quadratures for all retained modes are computed exactly using this single set of collocation points. At the same time, all modes that are retained for the computations are treated consistently. From Eq. (40) for the expression of the eigenvalues, it is easy to see that in order to perform the truncation in a physically consistent manner, we must retain

$$
K_{m}=|[K-|m| / 2]|,
$$

modes where the operation $|[\cdot]|$ denotes rounding down to the nearest integer. We note that by retaining such a varying number of modes in the radial direction for different angular momentum numbers $|m|$, we obtain, in the case with $\delta_{\alpha}=1$, that the highest order of the polynomial $R_{\alpha, m}$ is the same for different even values of $m$, or odd values of $m$ as can be seen from the expression given in Eq. (40). Using such a truncation, we can then consider two modes, one with $k=16$ and $m=0$ to obtain $2 k+|m|+1=33$, while for the second we have $k=13$ and $m=6$ so that $2 k+|m|+1=33$. We can see from Fig. 1(b), which shows these two modes corresponding to the same eigenvalues for different values of $m$, that the zeros of the $\varphi_{1,16}^{0}$ polynomial do span all the turning points of the $\varphi_{1,13}^{6}$ polynomial. We can, therefore, proceed by storing the values of the wavefunctions $R_{\alpha, m}$ at the zeros of $L_{K}\left(r^{2}\right)$.

The above procedure resolves the issue of how to choose the collocation points for a one component condensate. However, further complications arise in a two-component (or more generally multi-component) system when $\delta_{1} \neq \delta_{2}$. This is because the zeros of the Laguerre polynomials for the linear operator of the respective equations do not coincide which as clearly seen from the expression for $\varphi_{\alpha, k}^{m}$ in Eq. (35) is a function of $\delta_{\alpha}$. To integrate the system under the linear operator with spectral accuracy, we are forced to use a different set of zeros $\left\{r_{\alpha, i}\right\}$ for each component. However for practical pur- 
poses, we wish to store the values of the wavefunction at a single set of collocation points. Our approach will, therefore, be to store the values of the wavefunctions $\Phi_{\alpha}$ at the zeros of $L_{K}\left(r^{2}\right)$. According to Eq. (40), the effect of $\delta_{\alpha}$ is to stretch the spacial dependence of the eigenfunctions. This is clearly illustrated in Fig. 1(c) which shows that the zeros of the second component lie beyond the zeros of the first component for $\delta_{2}=1.667$. A different number of modes must, therefore, be retained for each component even for the same angular wavenumber. To determine the correct number of modes to retain in the second component, we replace Eq. (42) with

$$
K_{m}=\left|\left[\left((2 K+1) / \delta_{\alpha}-|m|-1\right) / 2\right]\right| .
$$

This leads to the desired property that the zeros of the retained modes do not extend beyond those corresponding to the collocation points defined above. This is clearly illustrated by the values of zeros shown in Fig. 1 (d) where for $m=6, k=16$, and $\delta_{2}=1.667$, we obtain $K_{m}=7$. We recall that, according to our definition, $\delta_{2} \geqslant 1$ and so we can guarantee that the zeros of the Laguerre polynomials for the second component always lie within the interval spanned by the zeros $\left\{r_{1, i}\right\}$ that are used to store the values of the wavefunctions.

A key question that we are now faced with is how to reconstruct the wavefunction at other desired values of $r$ and most importantly how to do so accurately and in a computationally efficient manner. In order to preserve the spectral-like accuracy of our scheme, we will reconstruct the wavefunction from the values at $r_{1, i}$ using the spectral representation of our scheme. Using Eqs. (31) and (34), we can evaluate $R_{2, m}$ using

$$
R_{2, m}\left(r_{1, i}\right)=\sum_{i=1}^{K} R_{2, m}\left(r_{2, i}\right)\left(\lambda_{2, i} \sum_{k=0}^{K_{0}-1} \varphi_{2, k}^{m^{*}}\left(r_{2, i}\right) \varphi_{2, k}^{m}\left(r_{1, i}\right)\right) .
$$

It follows that $R_{2, m}\left(r_{2, i}\right)$ can be obtained provided we can invert the matrix corresponding to the terms in the brackets appearing on the right-hand side of Eq. (44). This can easily be carried out through the use of a singular value decomposition (SVD).

Having defined $K_{m}$ and addressed how to reconstruct the values $R_{\alpha, m}$ at the respective collocation points $\left\{r_{\alpha, i}\right\}$ of each component, we can now proceed by expressing the reduced wavefunction in terms of Lagrange polynomials constructed from the generalised-Laguerre polynomial basis such that

$$
R_{\alpha, m}(r)=\sum_{i=1}^{K} R_{\alpha, m}\left(r_{\alpha, i}\right) f_{\alpha, i}(r)=\sum_{i=1}^{K} R_{\alpha, m}\left(r_{\alpha, i}\right)\left(\lambda_{i} \sum_{k=0}^{K_{m}-1} \varphi_{\alpha, k}^{m^{*}}\left(r_{\alpha, i}\right) \varphi_{\alpha, k}^{m}(r)\right)=\sum_{k=0}^{K_{m}-1} \tilde{R}_{\alpha, k m} \varphi_{\alpha, k}^{m}(r) .
$$

The advantage of expressing $R_{\alpha, m}$ in the above form is that once $\tilde{R}_{\alpha, k m}$ is known, the Laguerre transform can be performed directly owing to the orthogonality condition given in Eq. (41). The coefficients of the generalised-Laguerre polynomials can be evaluated very efficiently using matrix multiplication by writing

$$
\left(\begin{array}{c}
\tilde{R}_{\alpha, 0 m} \\
\tilde{R}_{\alpha, 2 m} \\
\vdots \\
\tilde{R}_{\alpha,(K-1) m}
\end{array}\right)=\left(\begin{array}{cccc}
\lambda_{\alpha, 1} \varphi_{\alpha, 0}^{m^{*}}\left(r_{1}\right) & \lambda_{\alpha, 2} \varphi_{\alpha, 0}^{m^{*}}\left(r_{2}\right) & \cdots & \lambda_{\alpha, K} \varphi_{\alpha, 0}^{m^{*}}\left(r_{K}\right) \\
\lambda_{\alpha, 1} \varphi_{\alpha, 1}^{m^{*}}\left(r_{1}\right) & \lambda_{\alpha, 2} \varphi_{\alpha, 1}^{m^{*}}\left(r_{2}\right) & \cdots & \lambda_{\alpha, K} \varphi_{\alpha, 1}^{m^{*}}\left(r_{K}\right) \\
\vdots & & \ddots & \vdots \\
\lambda_{\alpha, 1} \varphi_{\alpha,(K-1)}^{m^{*}}\left(r_{1}\right) & \lambda_{\alpha, 2} \varphi_{\alpha,(K-1)}^{m^{*}}\left(r_{2}\right) & \cdots & \lambda_{\alpha, K} \varphi_{\alpha,(K-1)}^{m^{*}}\left(r_{K}\right)
\end{array}\right)\left(\begin{array}{c}
R_{\alpha, m}\left(r_{\alpha, 1}\right) \\
R_{\alpha, m}\left(r_{\alpha, 2}\right) \\
\vdots \\
R_{\alpha, m}\left(r_{\alpha, K}\right)
\end{array}\right),
$$

which can be written in compact notation as

$$
\tilde{\mathrm{R}}_{\alpha, m}=\mathbf{T}_{\alpha} \mathrm{R}_{\alpha, m}
$$

Hence by convolving Eq. (30) with $\varphi_{\alpha, k}^{m^{*}}(r)$, and using the orthogonality condition (41), Eq. (30) then transforms to

$$
i \frac{d \tilde{R}_{\alpha, k m}}{d t}=[(2 k+|m|+1)-m \Omega] \tilde{R}_{\alpha, k m}
$$

which has the exact solution

$$
\tilde{R}_{\alpha, k m}\left(t_{n}^{*}\right)=\mathrm{e}^{-i[(2 k+|m|+1)-m \Omega]\left(t_{n}^{*}-t_{n}\right)} \tilde{R}_{\alpha, k m}\left(t_{n}\right) .
$$

We, therefore, see that the linear operator can be solved exactly using the above representation without the need to perform the additional splitting discussed in Section 3 between the angular and radial contributions respectively.

To summarise, the solution of the linear operator involves first Fourier transforming the reduced wavefunctions $\Phi_{\alpha}$ to obtain the quantities $R_{\alpha, m}\left(r_{1, i}\right)$. The evolution is then performed by carrying out the steps shown in Eq. (50), which is finally followed by a propagation of the nonlinear term in physical space.

$$
R_{\alpha, m}\left(r_{1, i}, t_{n}\right) \stackrel{1}{\longrightarrow} R_{\alpha, m}\left(r_{\alpha, i}, t_{n}\right) \stackrel{2}{\longrightarrow} \tilde{R}_{\alpha, k m}\left(t_{n}\right) \stackrel{[3}{\longrightarrow} \tilde{R}_{\alpha, k m}\left(t^{(1)}\right) \stackrel{\text { 4 }}{\longrightarrow} R_{\alpha, m}\left(r_{\alpha, i}, t^{(1)}\right) \stackrel{5}{\longrightarrow} R_{\alpha, m}\left(r_{1, i}, t^{(1)}\right) .
$$


Now focusing on Eq. (50), steps 5 and 1 correspond to the action given by Eqs. (44) together with the inverse transformation, respectively. In analogy with Eqs. (46) and (47) this can be written in matrix form as

$$
\mathrm{R}_{2, m}\left(r_{1, i}\right)=\mathbf{F R}_{2, m}\left(r_{2, i}\right)
$$

The matrix $\mathbf{F}$ has dimensions $K_{2,0} \times K_{1,0}$. To determine the vector $\mathrm{R}_{2, m}\left(r_{2, i}\right)$ which, given the interpolating property of the generalised-Laguerre polynomials, provide the values of the wavefunction at the points $r_{2, i}$, we must invert the matrix $\mathbf{F}$. However, the matrix $\mathbf{F}$ will in general not be a square matrix. Following our discussion of how to truncate the basis for different values of the angular wavenumber $m$, and the bounds we have on $\delta_{\alpha}$, we find that in general $K_{2, m} \leqslant K_{1, m}$. Therefore, inverting $\mathbf{F}$ requires performing a singular value decomposition (SVD) such that

$$
\mathbf{F}=\mathbf{L D R}^{T}
$$

where $\mathbf{R}$ is a $K_{2,0} \times K_{2,0}$ matrix, $\mathbf{D}$ is a diagonal $K_{2,0} \times K_{2,0}$ matrix, and $\mathbf{L}$ is a $K_{1,0} \times K_{2,0}$ matrix. The inverse can then be reconstructed as

$$
\mathbf{F}^{-1}=\mathbf{R D}^{-1} \mathbf{L}^{T}
$$

allowing straightforward calculation of the radial dependence of the angular components of the wavefunction given by $R_{2, m}\left(r_{2, i}\right)$. Once these values are known, step 2 is applied, which corresponds to the application of the orthogonality condition given in matrix form by Eq. (47), to reduce the evolution equation for $R_{\alpha, m}$ into the form given by Eq. (48). Step 3 evolves the modes $\tilde{R}_{\alpha, k m}$ exactly using Eq. (49). The propagated modal coefficients $\tilde{R}_{\alpha, k m}$ are then transformed back to coefficients $R_{\alpha, m}\left(r_{\alpha, i}\right)$ in step 4 by the inverse matrix $\mathbf{T}_{\alpha}^{-1}$. Being a square matrix, this can easily be computed. These values are subsequently interpolated to the collocation points $\left\{r_{1, i}\right\}$ by straightforward application of Eq. (51) to recover the coefficients $R_{2, m}\left(r_{1, i}, t^{(1)}\right)$.

\subsection{D Gross-Pitaevskii equation}

We now extend our pseudospectral method to the solution of the coupled Gross-Pitaevskii equations in 3D. As mentioned earlier, in contrast to other approaches, we will employ a spherical coordinate system which provides a more natural and efficient method of modelling the condensate. We begin by recalling that in order to perform the spherical harmonic transform (SHT), we represent the wavefunctions $\Phi_{\alpha}(r, \theta, \phi)$ at a set of discrete points given by $\Phi_{\alpha}\left(r_{i}, \theta_{j}, \phi_{s}\right)$ where $\theta_{j}=2 \pi j / M$, with $M$ taken to be an integer power of 2 such that $0 \leqslant j \leqslant M-1$, and $\phi_{s}$ are chosen as the zeros of the Legendre polynomial of degree $N=M / 2+1$. As before, the discretisation in the radial direction is denoted by an as yet, unspecified set of points $r_{\alpha, i}$ where $0 \leqslant i \leqslant K-1$. An SHT involves taking the convolution of Eq. (10) with the spherical harmonics $Y_{l}^{m}(\theta, \phi)$. The convolution in the $\theta$ direction amounts to a direct application of an FFT. The transform in the $\phi$ direction is carried out in an analogous manner to the generalised-Laguerre transform described in the previous section. In particular, our choice of collocation points in $\phi$ allows us to satisfy the orthogonality condition for the associated Legendre polynomials exactly for any polynomial of degree $\leqslant M / 2$. The equation corresponding to Eq. (41) for the associated Legendre polynomials then becomes

$$
\sum_{l=0}^{M / 2-1} \tilde{P}_{l}^{m}\left(\cos \phi_{s}\right) \tilde{P}_{l^{\prime}}^{m^{\prime}}\left(\cos \phi_{s}\right) w\left(\cos \phi_{s}\right)=\lambda_{s}^{-1} \delta_{l, l^{\prime}}
$$

where $\tilde{P}_{l}^{m}$ are the normalised associated Legendre polynomials so that $\tilde{P}_{l}^{m}=P_{l}^{m}[(2 l+1)(l-m) ! /(2(l+m) !)]^{1 / 2}$. With this, we can then setup the transform matrix in an identical manner to Eqs. (46) and (47). We note that no complications arise with the choice of collocation points in the $(\theta, \phi)$ directions thereby simplifying the formulation of the SHT transform even in the case of 2 component atomic condensates with different atomic masses. Hence, starting with Eq. (10) describing the evolution of the reduced wavefunctions $\Phi_{\alpha}$ under the linear operator, an application of an SHT reduces the equation to the form

$$
i \frac{\partial R_{\alpha, l m}\left(r_{\alpha, i}, t\right)}{\partial t}=\frac{-\delta_{\alpha}}{2} \frac{\partial^{2} R_{\alpha, l m}\left(r_{\alpha, i}, t\right)}{\partial r^{2}}+\frac{\delta_{\alpha} l(l+1) R_{\alpha, l m}\left(r_{\alpha, i}, t\right)}{2 r_{\alpha, i}^{2}}+\frac{r_{\alpha, i}^{2}}{2 \delta_{\alpha}} R_{\alpha, l m}\left(r_{\alpha, i}, t\right)-m \Omega_{z} R_{\alpha, l m}\left(r_{\alpha, i}, t\right),
$$

which describes the evolution of $R_{\alpha, l m}$ at the set of points $r_{\alpha, i}, \theta_{j}, \phi_{s}$. While an SHT is not as efficient as an FFT, we point out that other methods have been proposed that can carry out this transformation in a reduced number of operations [23]. However, in this work it will suffice to use a direct method since in our examples $M$ is not too large. Now proceeding as in the $2 \mathrm{D}$ case, we write $R_{\alpha, l m}(r, t)$ as

$$
R_{\alpha, l m}(r, t)=\sum_{i=1}^{K} R_{\alpha, l m}\left(r_{\alpha, i}, t\right) f_{\alpha, i}(r)
$$


where $f_{\alpha, i}(r)$ are Lagrange functions satisfying the condition $f_{\alpha, i}\left(r_{\alpha, j}\right)=\delta_{i j}$. Seeking a representation of $f_{\alpha, i}(r)$ in terms of an orthonormal basis of the linear operator of the 3D Gross-Pitaevskii equation leads to

$$
\varphi_{\alpha, k}^{l}(r)=C_{\alpha, k l} r^{(l+1)} \mathrm{e}^{-r^{2} /\left(2 \delta_{\alpha}\right)} L_{k}^{(l+1 / 2)}\left(\frac{r^{2}}{\delta_{\alpha}}\right)
$$

where the normalisation coefficient is given by

$$
C_{\alpha, k l}=\left[\frac{2^{2(k+l+1)}}{\delta_{\alpha}^{l+3 / 2} \pi^{1 / 2}}\right]^{1 / 2}\left[\frac{k !(k+l) !}{(2 k+2 l+1) !}\right]^{1 / 2} .
$$

As before, this basis can be used to simplify our evolution equation by making use of the corresponding eigenvalue equation for the generalised-Laguerre polynomials given by

$$
\left[-\frac{\delta_{\alpha}}{2} \frac{d^{2}}{d r^{2}}+\frac{\delta_{\alpha} l(l+1)}{2 r^{2}}+\frac{r^{2}}{2 \delta_{\alpha}}-m \Omega_{z}\right] \varphi_{\alpha, k}^{l}=\left[\left(2 k+l+\frac{3}{2}\right)-m \Omega_{z}\right] \varphi_{\alpha, k}^{l}, \quad k=0,1,2, \ldots
$$

The condition corresponding to Eq. (34) in 3D becomes

$$
\sum_{k=0}^{K_{l}-1} L_{k}^{(l+1 / 2)}\left(\frac{r_{\alpha, i}^{2}}{\delta_{\alpha}}\right) L_{k^{\prime}}^{(l+1 / 2)}\left(\frac{r_{\alpha, i}^{2}}{\delta_{\alpha}}\right) w\left(r_{\alpha, i}\right)=\lambda_{\alpha, i}^{-1} \delta_{k, k^{\prime}}
$$

where now $w(r)=r^{2(l+1)} \mathrm{e}^{-r^{2} / \delta_{\alpha}}$, and $K_{l}$ is an upper cut-off on the modes which depends on the angular wavenumber $l$. As before Eq. (60) can be computed exactly from $K$ collocation points provided that $r_{\alpha, i}$ are chosen as the zeros of the Laguerre polynomial $L_{K}^{1 / 2}\left(r^{2}\right)$ and an appropriate cut-off $K_{l}$ is imposed for polynomials of different order $l$. However, given that the energy spectrum is different from the $2 \mathrm{D}$ case, the cut-off we impose for the radial modes is defined differently. In particular, by referring to Eq. (59) that governs the energy spectrum of the linear radial operator, and proceeding as before for the $2 \mathrm{D}$ case by using the spectrum for the non-rotating case to determine the cut-off, we find that the modes must be truncated according to

$$
K_{l}=\left|\left[\frac{1}{2}\left(\delta_{\alpha}^{-1}\left(2 K+\frac{3}{2}\right)-l-\frac{3}{2}\right)\right]\right|
$$

As before, the operation $|[\cdot]|$ denotes rounding down to the nearest integer. With this choice of $K_{l}$, we have a consistent representation of all our modes and, in general, we find that condition (60) is satisfied exactly. In Fig. 2(a) and (b), we plot polynomials $\varphi_{1, k}^{l}$ of degree $k=13$ and $k=16$ for $l=0$ and $l=6$. As with the $2 \mathrm{D}$ case, we clearly see that if an equal number of modes is retained for different angular wavenumbers $l$, the zeroes of modes corresponding to $l>0$ would lie beyond those corresponding to $l=0$. In this case, an accurate representation of the wavefunction using the collocation points of the $l=0$ angular mode cannot be guaranteed. However, by truncating the spectrum of generalised-Laguerre polynomials according to Eq. (61), we see that all the zeroes of these polynomials with a higher angular wavenumber lie within the range spanned by zeroes of $l=0$.

In the case when $\delta_{2} \neq 1$, the zeroes for the Laguerre polynomials for the second component do not coincide with those of the first. An interpolation matrix is needed to reconstruct the values of $R_{2, l m}$ at the zeros of $L_{K}^{1 / 2}\left(r^{2} / \delta_{2}\right)$ so that the quadrature can be evaluated exactly for polynomials of degree $2 K-1$. In Fig. 2(c), we show how the zeroes of the Laguerre polynomials are shifted between the two components in the case when $\delta_{2} \neq 1$ (recall that $\delta_{2}>1$ based on our choice of non-dimensionalisation). In order to ensure the zeroes for the second component continue to lie within the interval spanned by the zeroes $\left\{r_{1, i}\right\}$, we must truncate the basis for the second component more severely as governed by Eq. (61). The results of such a truncation are illustrated in Fig. 2(d) which illustrates that we obtain the desired properties for the modes retained in our basis. It follows from these considerations that the evolution of the reduced wavefunction under the action of the 3D linear operator is reduced to steps similar to those presented by Eq. (50). As with the 2D problem, the entire set of operations can then be represented in terms of matrices.

We can now express the reduced wavefunction in terms of Lagrange polynomials constructed from the generalisedLaguerre polynomial basis such that

$$
R_{\alpha, l m}(r)=\sum_{i=1}^{K} R_{\alpha, l m}\left(r_{\alpha, i}\right) f_{\alpha, i}(r)=\sum_{i=1}^{K} R_{\alpha, l m}\left(r_{\alpha, i}\right)\left(\lambda_{\alpha, i} \sum_{k=0}^{K_{l}-1} \varphi_{\alpha, k}^{l^{*}}\left(r_{\alpha, i}\right) \varphi_{\alpha, k}^{l}(r)\right)=\sum_{k=0}^{K_{l}-1} \tilde{R}_{\alpha, k l m} \varphi_{\alpha, k}^{l}(r)
$$

The coefficients of the generalised-Laguerre polynomials can be evaluated very efficiently using matrix multiplication by writing 


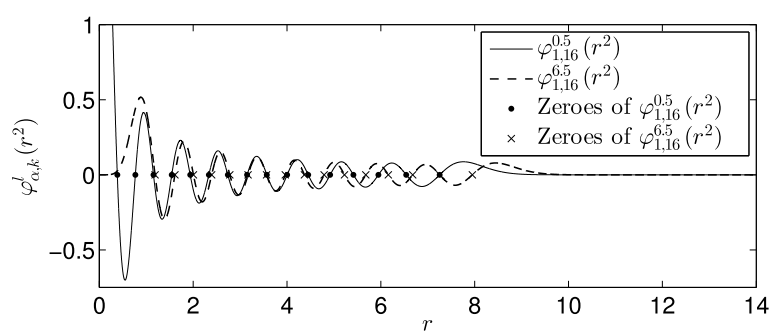

(a) Profiles and zeroes of polynomials for component $1\left(\delta_{1}=1\right)$ corresponding to $\varphi_{1,16}^{0.5}\left(r^{2}\right)$ and $\varphi_{1,16}^{6.5}\left(r^{2}\right)$.

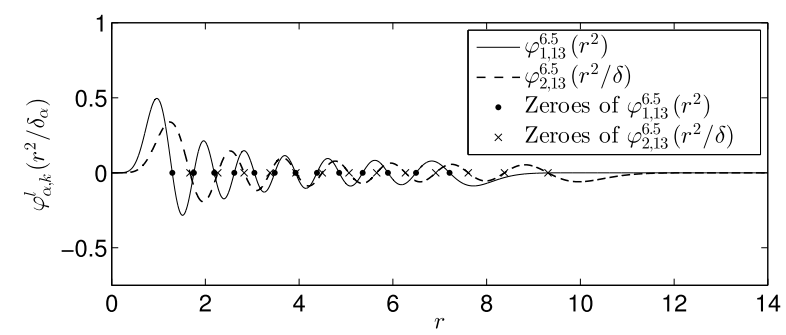

(c) Profiles and zeroes of polynomials for components $1\left(\delta_{1}=1\right)$ and $2\left(\delta_{2}=\delta=1.667\right)$ for $\varphi_{1,13}^{6.5}\left(r^{2}\right)$ and $\varphi_{2,13}^{6.5}\left(r^{2} / \delta\right)$.

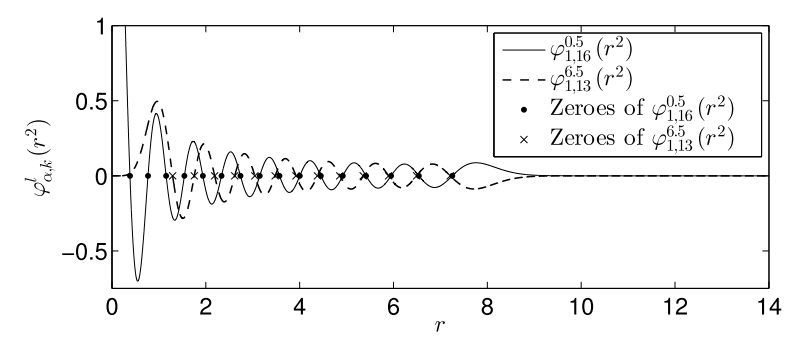

(b) Profiles and zeroes of polynomials for component $1\left(\delta_{1}=1\right)$ corresponding to $\varphi_{1,16}^{0.5}\left(r^{2}\right)$ and $\varphi_{1,13}^{6.5}\left(r^{2}\right)$.

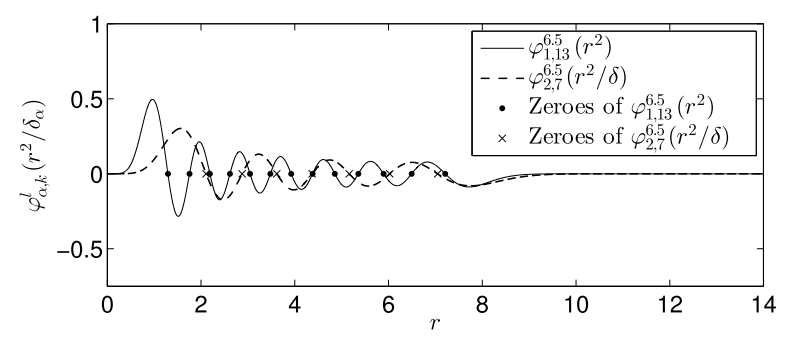

(d) Profiles and zeroes of polynomials for components $1\left(\delta_{1}=1\right)$ and $2\left(\delta_{2}=\delta=1.667\right)$ for $\varphi_{1,13}^{6.5}\left(r^{2}\right)$ and $\varphi_{2,7}^{6.5}\left(r^{2} / \delta\right)$.

Fig. 2. Comparison of different generalised-Laguerre polynomials $\varphi_{\alpha, k}^{l+0.5}\left(r^{2} / \delta_{\alpha}\right)$ of degree $k$ and order $l$ for component $\alpha$ in 3D.

$$
\left(\begin{array}{c}
\tilde{R}_{\alpha, 0 l m} \\
\tilde{R}_{\alpha, 2 l m} \\
\vdots \\
\tilde{R}_{\alpha,(K-1) l m}
\end{array}\right)=\left(\begin{array}{cccc}
\lambda_{\alpha, 1} \varphi_{0}^{*}\left(r_{1}\right) & \lambda_{\alpha, 2} \varphi_{0}^{*}\left(r_{2}\right) & \cdots & \lambda_{\alpha, K} \varphi_{0}^{*}\left(r_{K}\right) \\
\lambda_{\alpha, 1} \varphi_{1}^{*}\left(r_{1}\right) & \lambda_{\alpha, 2} \varphi_{1}^{*}\left(r_{2}\right) & \cdots & \lambda_{\alpha, K} \varphi_{1}^{*}\left(r_{K}\right) \\
\vdots & & \ddots & \vdots \\
\lambda_{\alpha, 1} \varphi_{(K-1)}^{*}\left(r_{1}\right) & \lambda_{\alpha, 2} \varphi_{(K-1)}^{*}\left(r_{2}\right) & \cdots & \lambda_{\alpha, K} \varphi_{(K-1)}^{*}\left(r_{K}\right)
\end{array}\right)\left(\begin{array}{c}
R_{\alpha, l m}\left(r_{\alpha, 1}\right) \\
R_{\alpha, l m}\left(r_{\alpha, 2}\right) \\
\vdots \\
R_{\alpha, l m}\left(r_{\alpha, K}\right)
\end{array}\right)
$$

which can be written in compact notation as

$$
\tilde{\mathrm{R}}_{\alpha, l m}=\mathbf{T R}_{\alpha, l m} .
$$

Hence by convolving the above equation with $\varphi_{\alpha, k}^{l *}$, and using the orthogonality condition (60), Eq. (55) then transforms to

$$
i \frac{d \tilde{R}_{\alpha, k l m}}{d t}=\left[\left(2 k+l+\frac{3}{2}\right)-m \Omega_{z}\right] \tilde{R}_{\alpha, k l m}
$$

which has the exact solution

$$
\tilde{R}_{\alpha, k l m}\left(t_{n}^{*}\right)=\mathrm{e}^{-i\left[(2 k+l+3 / 2)-m \Omega_{z}\right]\left(t_{n}^{*}-t_{n}\right)} \tilde{R}_{\alpha, k l m}\left(t_{n}\right) .
$$

\subsection{Efficient implementation of the pseudospectral scheme}

At first glance, the method we have presented appears inefficient requiring interpolation matrices and inversion or even singular value decomposition (SVD) at every step. However, these computations can all be performed as a single preprocessing step and combined into a single matrix propagator. We first observe that the transformation matrix $\mathbf{F}$ that is used to interpolate $R_{\alpha, m}\left(r_{1, i}\right)$ to $R_{\alpha, m}\left(r_{\alpha, i}\right)$ and its inverse $\mathbf{F}^{-1}$ are independent of time. In fact, once the truncation has been specified, these matrices are fixed throughout the remainder of the computation. We, therefore, evaluate the matrices $\mathbf{F}$ and $\mathbf{F}^{-1}$, and the transformation matrices $\mathbf{T}$ and $\mathbf{T}^{-1}$ at the beginning of the computation as a preprocessing step. The evolution of $R_{\alpha, m}\left(r_{1, i}\right)$ in 2D as schematically illustrated in Eq. (50) can then be written in matrix form as

$$
\tilde{\mathrm{R}}_{\alpha, m}\left(\mathbf{r}_{1}, t^{*}\right)=\mathbf{F T e} \mathrm{i}^{i, m}\left(t^{*}-t\right) \mathbf{T}^{-1} \mathbf{F}^{-1} \mathbf{R}_{\alpha, m}\left(\mathbf{r}_{1}, t\right),
$$

where $\mathbf{D}_{\alpha, m}$ is the matrix corresponding to the eigenvalues given by Eq. (40). Similarly the evolution of the vector $R_{\alpha, l m}\left(r_{1, i}\right)$ in $3 \mathrm{D}$ is given by

$$
\tilde{\mathrm{R}}_{\alpha, l m}\left(\mathbf{r}_{1}, t^{*}\right)=\mathbf{F} \mathbf{T e} \mathrm{i}^{i, l m}\left(t^{*}-t\right) \mathbf{T}^{-1} \mathbf{F}^{-1} \mathbf{R}_{\alpha, l m}\left(\mathbf{r}_{1}, t\right),
$$


where $\mathbf{D}_{\alpha, m}$ is the matrix corresponding to the eigenvalues given by Eq. (59). Starting from the end of Eqs. (67) and (68), we see that $\mathbf{F}^{-1} \mathrm{R}(t)$ corresponds to step 1 in the schematic shown in Eq. (50). The following multiplication then corresponds to 2 with subsequent matrices corresponding to operations proceeding progressively along the evolution outlined in Eq. (50). Now for a fixed time step $\Delta t$, we can simplify the entire evolution to a single matrix-vector multiplication given by

$$
\begin{aligned}
& \mathrm{R}\left(t^{*}\right)=\mathbf{P R}(t), \\
& \mathbf{P} \equiv \mathbf{F T e} e^{i \mathbf{D}\left(t^{*}-t\right)} \mathbf{T}^{-1} \mathbf{F}^{-1} .
\end{aligned}
$$

In the above equation, the vectors $\mathbf{R}$ and the matrix $\mathbf{D}$ would be indexed by the respective subscripts depending on whether we're dealing with the $2 \mathrm{D}$ or $3 \mathrm{D}$ case. This very simple means of evolving the wavefunction under the action of the linear operator retains the spectral accuracy provided by our method and is very efficient to implement either in MATLAB, which has been optimised for matrix-matrix and matrix-vector operations, or using standard libraries such as BLAS. Given our ability to formulate the scheme in this way, all the results presented in the next section were obtained by implementing the method on MATLAB. We note that the above operation for evaluating $R$ is the most costly per time step and for a direct computation requires $\mathcal{O}\left(M K^{2}\right)$ operations. The Fourier transform is $\mathcal{O}(K M \log M)$ in 2D. In 3D the spherical harmonic transform can be evaluated using more advanced methods to speed up the computation but a direct evaluation as used in this work requires $\mathcal{O}\left(K \mathrm{M}^{4}\right)$ (note that, based on our definitions, in 3D the number of points in the angular direction scale as $M^{2}$ ). The evaluation of the nonlinear terms is local in physical space and so when advancing the wavefunction in time under the nonlinear operator the cost is simply $\mathcal{O}(K M)$ in 2D and $\mathcal{O}\left(K M^{2}\right)$ in 3D. In terms of memory, the most significant costs arise from the need to store the matrix $\mathbf{P}$ for the generalised-Laguerre transform and the memory costs associated with the FFT and SHT which depend on the scheme used.

\section{Results}

Having presented an efficient and highly accurate method for evolving our wavefunction, we will now illustrate the method with a number of examples. We will begin by benchmarking our numerical scheme against some standard test cases for a condensate in a harmonic trap. Our first test case corresponds to the Goss-Pitaevskii equation with no interaction potentials such that the equation reduces to the linear Schrödinger with an external trap but with no rotation. This allows us to derive analytical solutions permitting a detailed quantitative check of the numerical accuracy of our method. In particular, we will demonstrate that our method does indeed recover spectral convergence in space and is second order accurate in time. Since the 2D and 3D numerical schemes are closely related, we will focus on the 2D problem for these numerical studies. We begin by noting that the solution of the wavefunction $\psi$ for a single component system in a harmonic external potential but with a non-zero residual potential $V_{\text {res }}=\lambda_{y} y^{2} / 2$ can be expressed as

$$
\begin{aligned}
& \psi_{\text {anal }}(x, y) \\
& =\sum_{n_{x}, n_{y}} c\left(n_{x}, n_{y}\right)\left(\frac{\sqrt{\lambda_{y, \alpha}}}{\pi \delta_{\alpha}}\right)^{1 / 2} \frac{1}{\sqrt{2^{n_{x}+n_{y}} n_{x} ! n_{y} !}} H_{n}\left(\frac{x}{\sqrt{\delta_{\alpha}}}, \frac{y}{\sqrt{\lambda_{y, \alpha} \delta_{\alpha}}}\right) \mathrm{e}^{-\left(x^{2}+\lambda_{y, \alpha} y^{2}\right) / 2 \delta_{\alpha}} \mathrm{e}^{-i\left[\left(n_{x}+1 / 2\right)+\left(n_{y}+1 / 2\right) \lambda_{y, \alpha}\right] t},
\end{aligned}
$$

where $H_{n}$ is the Hermite polynomial of order $n$ and $c\left(n_{x}, n_{y}\right)$ is a weight factor associated with each mode. In the numerical results to be presented here, we have set the sum to include the modes $n_{x}=1,2$ and $n_{y}$ to vary from 1 to 10 . We set all the modes with equal weighting and in order to ensure $\psi_{\text {anal }}$ normalises to unity, we set $c\left(n_{x}, n_{y}\right)=1 / \sqrt{20}$ for these twenty modes and zero otherwise. To test the spectral convergence of the spatial discretisation, we set $\delta_{\alpha}=1, \lambda_{y, \alpha}=1$, and evaluated the error in calculating the partial derivative $\partial \psi / \partial_{x}=\partial \psi / \partial_{r} \cos (\theta)-\psi / \partial_{\theta} \sin (\theta) / r$. Clearly, this requires accurate evaluation of the derivatives in the circumferential and radial directions. We computed the norm $\left\|\psi-\psi_{\text {anal }}\right\|_{\infty}$ in evaluating the derivative by varying the total number of grid points (equivalently modes) while keeping the number of radial modes equal to the number of azimuthal modes. Fig. 3(a) shows that the error gradually decreases but then falls sharply at around $K=23$. This occurs because our solution is bandwidth limited and so the spectral accuracy is achieved once a sufficient number of modes is used. To demonstrate the temporal accuracy of our scheme, we now set $\lambda_{y, \alpha}^{2}=1$.44. In our numerical integration this introduces a residue external potential which now introduces a splitting error from the time integration. We integrated the initial condition corresponding to the analytical expression from $t=0$ to $t=1$ and computed the infinity norm at the end of the time integration. The results obtained for different time steps is presented in Fig. 3(b). The curve $0.05 t^{-2}$ has been included as a reference to illustrate the second order convergence in time of our scheme.

We will now consider a fully nonlinear problem by calculating the ground state of a single component wavefunction in cylindrically and spherically symmetric traps without rotation. To compute the ground states, we have used the imaginary time evolution as described at the end of Section 3. Starting with an initial condition corresponding to a Gaussian density profile on a grid of $(r, \theta)=(96 \times 64)$ in $2 \mathrm{D}$, and $(r, \theta, \phi)=(96 \times 64 \times 32)$ in 3D, we marched the equations forward in time for the case $\Omega_{z}=0$ until the wavefunction converged to the steady state as shown in Fig. 4. The results presented were obtained using a time step $\Delta t=0.001$. To verify our numerical scheme, we have compared our numerical predictions against 


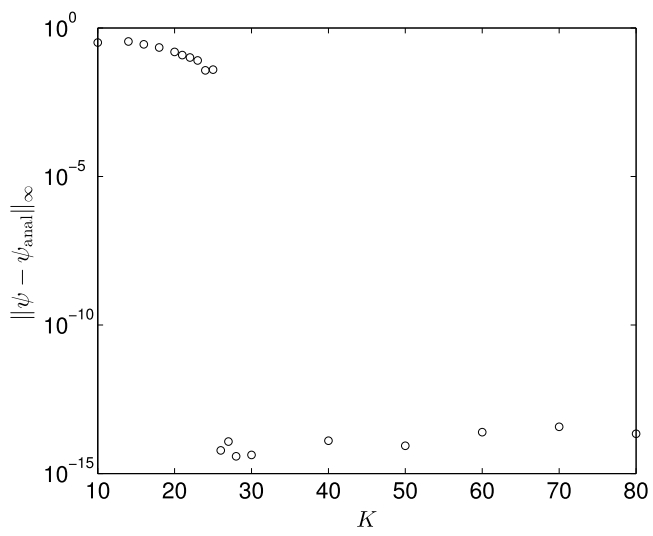

(a) Spatial convergence.

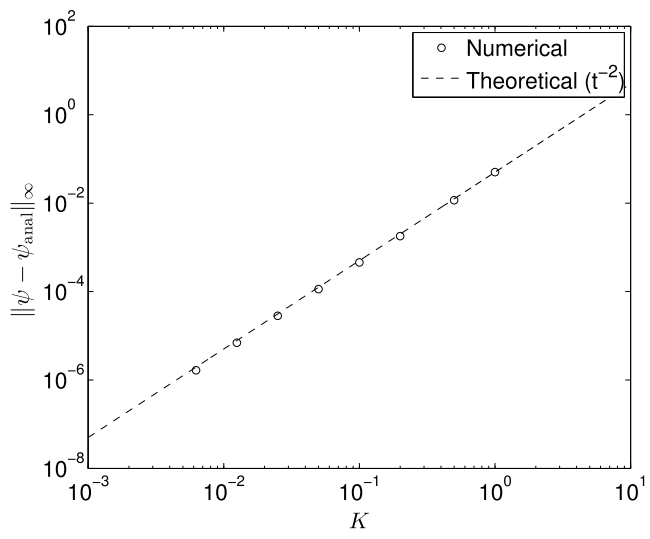

(b) Temporal convergence.

Fig. 3. Variation of the infinity norms for spatial and temporal convergence for 2D case; (a) spatial convergence as function of number of grid points (number of radial and azimuthal grid modes was equal, i.e. $K=M$ ), (b) temporal convergence as a function of time step $\Delta t$ compared against $t^{-2}$ curve.

the recent theoretical results of Salman [44] where a divergence-free WKB method [31] was developed to obtain asymptotic results for this problem. The theoretical approximation has the advantage that it is uniformly valid describing the transition of the wavefunction from the classically allowed region occurring at smaller values of $r$, where the Laplacian term is negligible, all the way into the classically forbidden region where the kinetic energy becomes important. The healing layers (i.e. regions where quantum corrections become significant) are, therefore, represented in this theoretical approximation in contrast to the commonly used Thomas-Fermi profile [42]. In the case of the latter, the kinetic energy term is neglected altogether. The asymptotic results of [44] allow a more thorough validation of our numerical scheme. From [44], the profile of the condensate can be approximated by setting

$$
\psi(x, y, z)=\exp \left[\int \varphi^{\prime} d x+\int \vartheta^{\prime} d y+\int \phi^{\prime} d z\right]
$$

and expanding the exponents, $\varphi^{\prime}$ in a power series given by $\varphi^{\prime}=\sum_{n=0}^{\infty} \epsilon^{2 n} \varphi_{n}^{\prime}$ and similarly for $\vartheta^{\prime}$ and $\phi^{\prime}$. The small parameter $\epsilon^{2}=\left(a_{h o} / R_{c}\right)^{4}$, where $R_{c}$ is the radius of the condensate, can be estimated as in [42] to obtain $R_{c}=a_{h o}\left(15 N a_{s} / a_{h o}\right)^{1 / 5}$. Upon substituting into the GP equation for a single component with a harmonic trap, we find that, at leading order, $\varphi_{0}^{\prime}$ is the real solution of the following cubic algebraic equation

$$
\varphi_{0}^{\prime 3}+\varphi_{0}^{\prime}\left[2\left(\mu-\frac{x^{2}}{2}\right)\right]+\epsilon \chi=0,
$$

where $\mu_{c}=\mu+\epsilon\left[\vartheta^{\prime \prime}(0)+\phi^{\prime \prime}(0)\right] / 2$. It follows that to leading order, the exponent is given by

$$
\begin{aligned}
\int_{0}^{x} \varphi_{0}^{\prime} d x^{\prime}= & x \varphi_{0}^{\prime}-\int_{0}^{x} x^{\prime} d \varphi_{0}^{\prime}=x \varphi_{0}^{\prime}-\frac{\epsilon}{2} \ln \left|\varphi_{0}^{\prime}\right|+\frac{1}{4}\left[\epsilon^{2}+8 \mu_{c} \varphi_{0}^{\prime 2}+4 \varphi_{0}^{\prime 4}\right]^{1 / 2} \\
& +\frac{\mu_{c}}{2}\left\{\ln \left[2\left(\mu_{c}+\varphi_{0}^{\prime 2}\right)+\left(\epsilon^{2}+8 \mu_{c} \varphi_{0}^{\prime 2}+4 \varphi_{0}^{\prime 4}\right)^{1 / 2}\right]\right\} \\
& -\frac{\epsilon}{4} \operatorname{arctanh}\left\{\left(\epsilon^{2}+4 \mu_{c} \varphi_{0}^{\prime 2}\right) /\left[\epsilon\left(\epsilon^{2}+8 \mu_{c} \varphi_{0}^{\prime 2}+4 \varphi_{0}^{\prime 4}\right)^{1 / 2}\right]\right\}-\varphi_{0}(0) .
\end{aligned}
$$

Similar expressions follow for $\vartheta^{\prime}$ and $\phi^{\prime}$. The next order in the expansion of the exponent is given by

$$
\varphi_{1}^{\prime}=\frac{\epsilon^{2}}{2\left(3 \epsilon^{2} \varphi_{0}^{\prime 2}+\epsilon^{2}\left[2\left(\mu_{c}-\tilde{V}_{1}\right)\right]\right)} \varphi_{0}^{\prime \prime \prime} .
$$

While the integral of this first order term for $\varphi_{1}^{\prime}$ cannot be obtained in closed form, we can evaluate it numerically to obtain an improved approximation to our leading order asymptotic expression. Comparisons between these asymptotic approximations and our numerical solutions are presented in Fig. 4(a) and (b) for the 2D and 3D cases, respectively. In the 2D case, the simulations were obtained for a gas consisting of $N=20,000{ }^{87} \mathrm{Rb}$ atoms which have an $s$-wave scattering length of $5.82 \times 10^{-9} \mathrm{~m}$, and a mass $m=1.44 \times 10^{-25} \mathrm{~kg}$ and confined in an in-plane isotropic trap of frequency $\omega_{\text {ho }}=$ $50 \mathrm{~Hz}$. On the other hand, the 3D simulation was computed assuming $N=250,000{ }^{87} \mathrm{Rb}$ atoms confined in an isotropic trap of frequency $\omega_{h o}=50 \mathrm{~Hz}$. These parameters lead to condensates with chemical potentials of $\mu=33.0482$ and $\mu=23.0577$, in $2 \mathrm{D}$ and $3 \mathrm{D}$ respectively. As can be seen from the figure, the comparison between the computed profile and the theoretical 


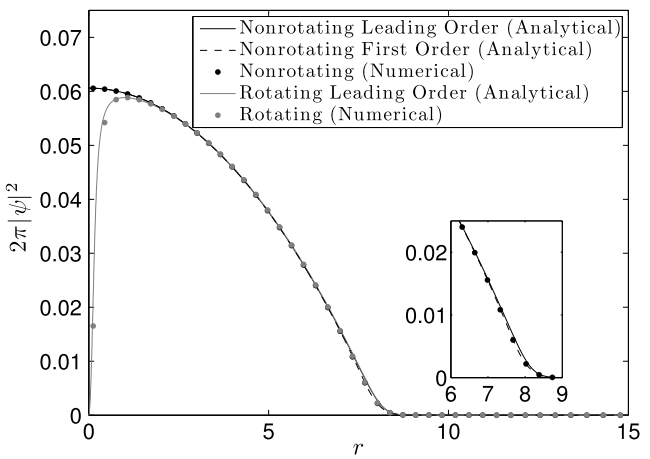

(a) Numerical and analytical solutions of condensate in $2 \mathrm{D}$ corresponding to $\mu=33.0482$; inset shows healing layer region.

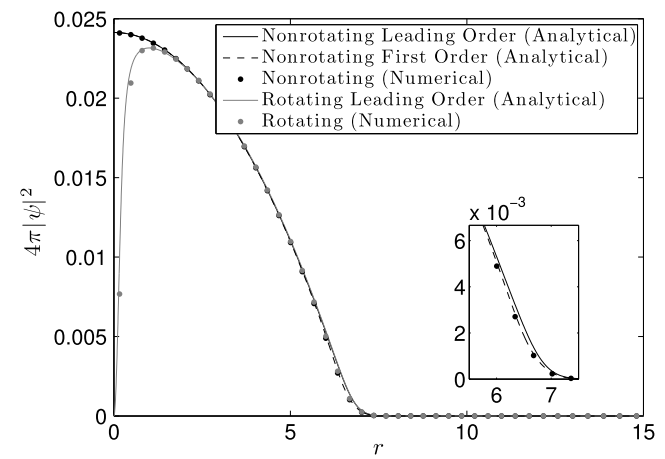

(b) Numerical and analytical solutions of condensate in 3D corresponding to $\mu=23.0577$; inset shows healing layer region.

Fig. 4. Comparison of analytical and numerical condensate profiles for (a) 2D condensate, (b) 3D condensate, with and without a single quantised vortex located at the centre of the condensate for a system consisting of $20,000{ }^{87} \mathrm{Rb}$ atoms in an isotropic trap with $\omega_{\text {ho }}=50 \mathrm{~Hz}$.

result is excellent over the entire interval of the computational domain. We note that while some discrepancies exist near the edge of the condensate, this stems from the asymptotic approximation used to obtain our theoretical results. Indeed, by retaining the correction from the next order in the asymptotic expansion, we see that the numerical predictions and theoretical results become indistinguishable.

The results presented in Fig. 4 demonstrate our scheme correctly predicts the ground state for the $l=0$ modes. However, a key feature that must be benchmarked is that the scheme works correctly for $l \neq 0$. This would then demonstrate that the time marching scheme given by Eqs. (67)-(68) which rely on the use of the transform matrices T, F between different grid points yields the correct results. For this purpose, we have repeated a similar ground state computation as in the above case with $\Omega_{z}=0$ but now we phase imprint a vortex onto our condensate solutions that are located at the centre of the traps. The vortex profile is obtained from the Padé approximant evaluated by Berloff [14]. After relaxing the solution through an imaginary time evolution of the GP equations, we find that the system settles into a metastable state consisting of a single vortex located at the centre of the traps. The vortex breaks the spherical symmetry thereby allowing us to check that the transfer matrices have been implemented correctly. A ground state computation for this problem is also shown in Fig. 4(a) and (b) for both 2D and 3D cases. The characteristic dip in the densities of the condensates at the centres of the traps seen for the rotating cases are indicative of the signature of a quantised vortex. As in the non-rotating case, an asymptotic approximation for the condensates using the divergence-free WKB method was also derived in [44]. This is now given by

$$
\begin{aligned}
\tilde{\varphi}(r)= & r \log (r) \tilde{\varphi}^{\prime}+\frac{r \tilde{\varphi}^{\prime}}{2}-\frac{1}{2} \epsilon \log \left(\left|r \tilde{\varphi}^{\prime}+\epsilon\right|\right)-\frac{1}{2} \log \left(\left|\frac{\mu r \tilde{\varphi}^{\prime}-\sqrt{\left(r \tilde{\varphi}^{\prime}\right)^{4}+\left(\mu^{2}-2 \epsilon^{2}\right)\left(r \tilde{\varphi}^{\prime}\right)^{2}+\epsilon^{4}}}{r \tilde{\varphi}^{\prime}-\epsilon}\right|\right) r \tilde{\varphi}^{\prime} \\
& -\frac{\epsilon}{2} \mathcal{R}\left\{\arctan \left(\frac{\mu\left[\left(r \tilde{\varphi}^{\prime}\right)^{2}+\epsilon^{2}\right]}{2 \epsilon \sqrt{\left(r \tilde{\varphi}^{\prime}\right)^{4}+\left(\mu^{2}-2 \epsilon^{2}\right)\left(r \tilde{\varphi}^{\prime}\right)^{2}+\epsilon^{4}}}\right)\right\} \\
& +\frac{\mu}{4} \log \left(\left|\frac{\mu^{2}}{2}-\epsilon^{2}+\left(r \tilde{\varphi}^{\prime}\right)^{2}+\sqrt{\left(r \tilde{\varphi}^{\prime}\right)^{4}+\left(\mu^{2}-2 \epsilon^{2}\right)\left(r \tilde{\varphi}^{\prime}\right)^{2}+\epsilon^{4}}\right|\right),
\end{aligned}
$$

where $\mathcal{R}(\cdot)$ denotes the real part. As before, we observe excellent agreement between the theory and the numerical results. These benchmarks clearly demonstrate the correct implementation of our numerical scheme.

We will now proceed by demonstrating our scheme for ground state computations of a vortex lattice beginning from a condensate without any vortices under the influence of rotation. We solved Eq. (2) for a single component in a toroidal trap by setting $\gamma_{12}=0$. As before, a grid of $(r, \theta)=(96 \times 64)$ was used and the equation was integrated in imaginary time with $\Delta t=0.001$ and for two different rotation speeds corresponding to $\Omega=0.225$ and $\Omega=0.5$ which are based on the parameters used in [2]. To break the symmetry and simulate the formation of a vortex lattice, we used a numerically computed ground state profile without vortices and imposed small perturbations on the condensate density. After evolving the system in time, we observed the formation of quantised vortices which settled into the patterns shown in Figs. 5 and 6. For slow rotation speeds, the vortices enter from the edges of the condensate and adopt a steady pattern. For faster rotation speeds, the toroidal trap modifies the density profile of the condensate to a ring-shaped condensate with vortices uniformly distributed along the azimuthal direction. While our results are similar to the results presented in [2], we nevertheless notice some differences from their results (see Figs. 4b and $4 \mathrm{~d}$ in [2]). These differences are possibly caused by the breaking of the azimuthal symmetry by the Cartesian grid used in [2]. Moreover, since the ground states are not necessarily unique, or may be nearly degenerate in the sense that several metastable states can be located very close to the ground state of the system, it is easy for the system to converge to slightly different solutions even for the same set of parameters.

Having illustrated our method for a one component system, we will now consider a two-component condensate. It has been shown that for components consisting of different atomic species, the difference in the atomic masses between 


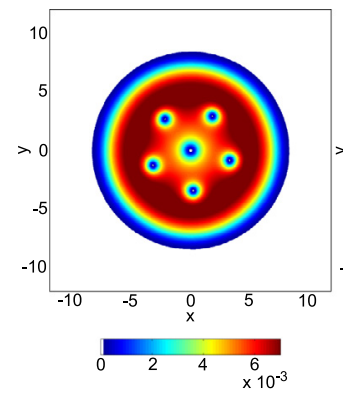

(a) Density

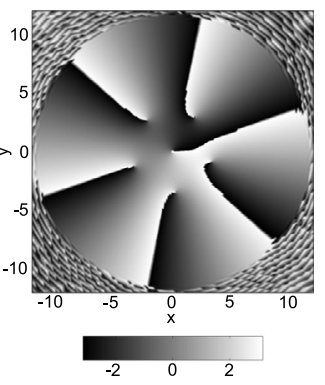

(b) Phase

Fig. 5. Density and phase contour plots of a rotating condensate in an isotropic harmonic trap with $\gamma^{(2 \mathrm{D})}=500, \lambda_{\mathrm{tr}}=60, l_{\mathrm{tr}}=0.1, \Omega_{z}=0.225$.

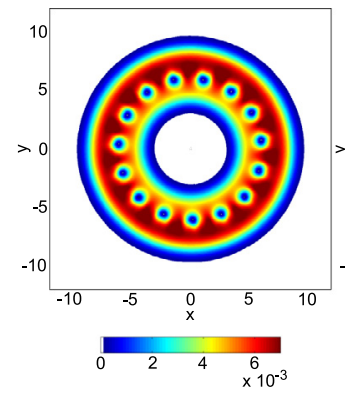

(a) Density

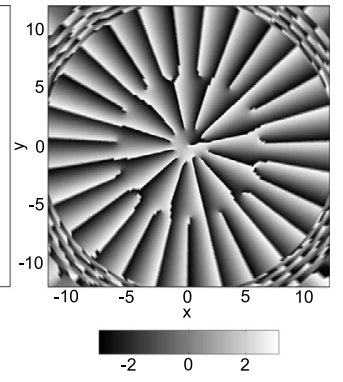

(b) Phase

Fig. 6. Density and phase contour plots of a rotating condensate in an isotropic harmonic trap with $\gamma^{(2 \mathrm{D})}=500, \lambda_{\mathrm{tr}}=60, l_{\mathrm{tr}}=0.1, \Omega_{z}=0.5$.

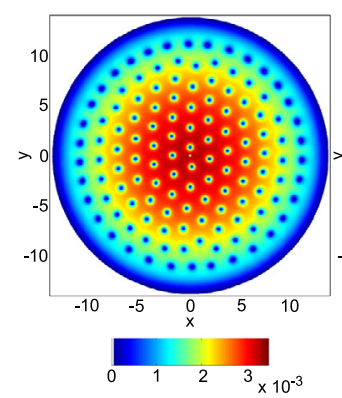

(a) Density of component 1

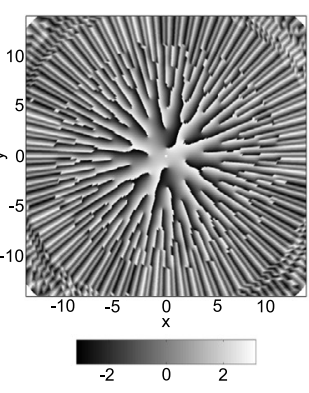

(b) Phase of component 1 .

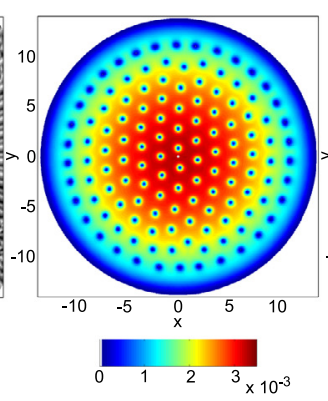

(c) Density of component 2 .

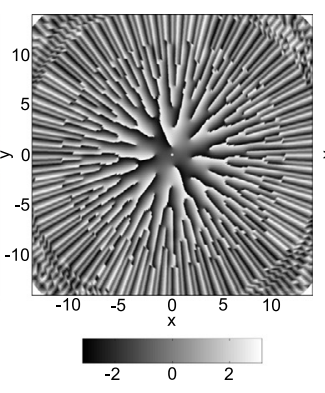

(d) Phase of component 2.

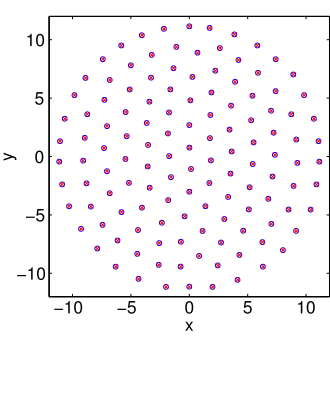

(e) Vortex lattices

Fig. 7. Vortex lattices in each component together with plot of superposition of vortex lattices in both components for $m_{1} / m_{2}=1.0$.

the species can lead to a ground state in which the vortices are distributed differently in each component from the case when the components are assumed to have equal masses (e.g. in the case of a system made up of atoms in different hyperfine states). The 2D vortex lattices in this case were first predicted in the work of [10] and later illustrated with numerical simulations of the GP equation in [11]. However, as discussed throughout the paper, this case raises additional challenges to simulate with the generalised-Laguerre basis functions since the collocation points for each component do not coincide under such situations. We will, therefore, present simulations for a two-component condensate to demonstrate that the method we have developed leads to an accurate and efficient scheme that can be used to simulate such systems. In direct analogy with the results of [11], we have considered a two-component condensate with $\gamma_{11}^{(2 \mathrm{D})}=2 \times 10^{4}$ and $\gamma_{12}^{(2 \mathrm{D})}=-2 \gamma_{11}^{(2 \mathrm{D})} / 3$. The number of atoms was assumed to be equal in each component so that $N_{1}=N_{2}$ and the angular rotation was set to $\Omega_{z}=0.9$. Two different mass ratios were considered, the first with $m_{1} / m_{2}=1.0$ and the second with $m_{1} / m_{2}=1.4$. After integrating the equations in imaginary time on a grid consisting of $(96 \times 128)$ collocation points in $(r, \theta)$, with a time step of $\Delta t=0.002$, we obtained the solutions presented in Figs. 7 and 8 . As can clearly be seen, for a mass ratio of unity the vortex lattices in both components are locked together as expected. However, when the mass ratio is increased from 1.0 to 1.4 , and the vortex lattice obtained in the former case is relaxed under further imaginary time evolution, we observe that the vortex lattices remain locked together within the inner regions of the condensate. On the outer regions, the vortices necessarily occupy different positions in space which is fully consistent with the analysis and results presented in $[10,11]$. The numerical simulations we have presented, therefore verify our numerical scheme even for the case of a multi-component condensate with different mass ratios. 


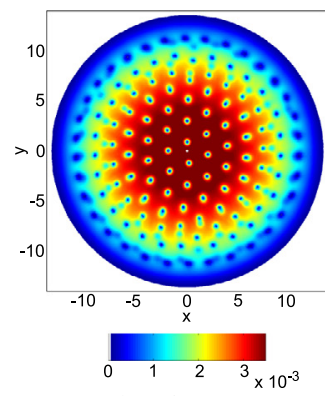

(a) Density of component 1 .

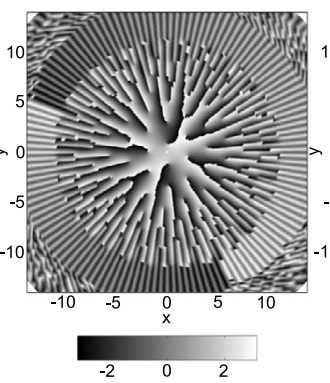

(b) Phase of component 1.

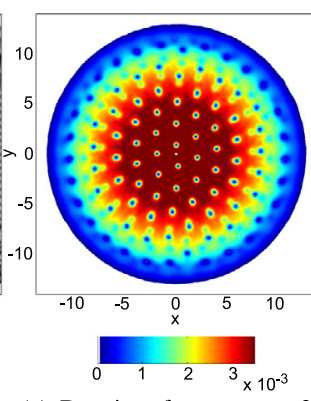

(c) Density of component 2 .

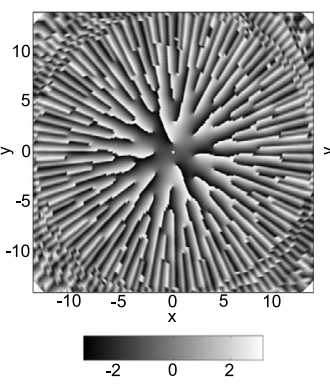

(d) Phase of component 2 .

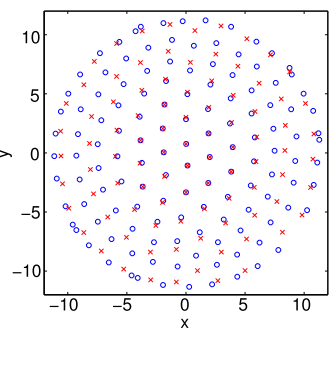

(e) Vortex lattices.

Fig. 8. Vortex lattices in each component together with plot of superposition of vortex lattices in both components for $m_{1} / m_{2}=1.4$.

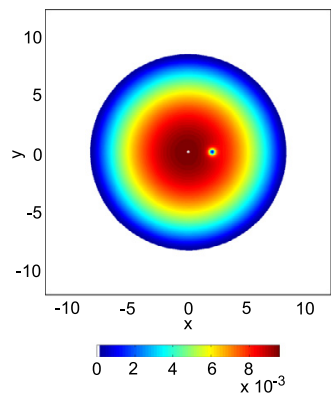

(a) Density at $t=0$.

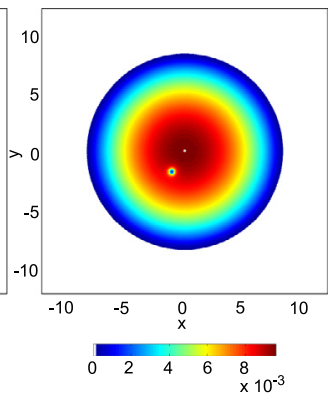

(b) Density at $t=46.72$.

Fig. 9. Density of a $2 \mathrm{D}$ condensate with a precessing quantised vortex located off-centre at a radius $r=2$ for a system consisting of $20,000{ }^{87} \mathrm{Rb}$ atoms in an isotropic trap with $\omega_{\text {ho }}=50 \mathrm{~Hz}$, and $\lambda_{\mathrm{tr}}=0, \Omega_{z}=0$.

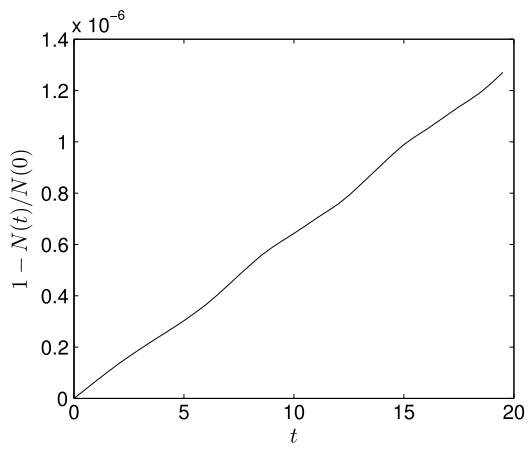

(a) L2 norm dependence on time.

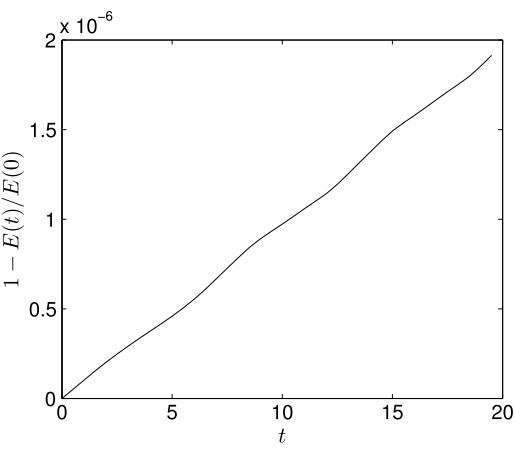

(b) Energy dependence on time.

Fig. 10. Conservation of L2 and energy norms for case of a $2 \mathrm{D}$ system consisting of $20,000{ }^{87} \mathrm{Rb}$ atoms in an isotropic trap with $\omega_{h o}=50 \mathrm{~Hz}$, and $\lambda_{\operatorname{tr}}=0, \Omega_{z}=0$.

Having demonstrated that our method correctly reproduces the ground state properties of a one and two-component system, we will now focus on time-dependent simulations of the GP system of equations. We begin by considering the precession of a single quantised vortex imprinted onto the ground state density of a non-rotating $2 \mathrm{D}$ condensate with parameters similar to those considered above for the results presented in Fig. 4(a). The ground state is computed numerically on a grid of $96 \times 64$ grid points in the $(r, \theta)$ directions, respectively, using the imaginary time evolution as described above. The vortex is then imprinted at $(r, \theta)=(2,0)$ by setting $\psi=\psi_{\mathrm{gs}} \psi_{\text {vortex }}$ where $\psi_{\mathrm{gs}}$ is the numerically computed ground state and $\psi_{\text {vortex }}$ is set to correspond to a vortex according to the specification given in [14] but where the core radius has been modified to account for the different condensate density at the position of the vortex. Using this initial condition, the wavefunction is evolved forward in time using the second order Strang splitting with a time step of $\Delta t=0.0005$. As can be seen in Fig. 9, the vortex precesses around the centre of the trap at a fixed radius as expected. Throughout the evolution, we have tracked both the L2 norm of $\psi$ (i.e. the total number of particles, $N$ ) and the total energy given by $H$. As can be seen in Fig. 10(a) and (b), both quantities are conserved to a high degree of accuracy throughout the time of integration. However due to our need to truncate the modes based on a uniform energy cut-off, our scheme does not conserve these quantities at the discretised level thereby resulting in the small variations that are seen in the figures. 


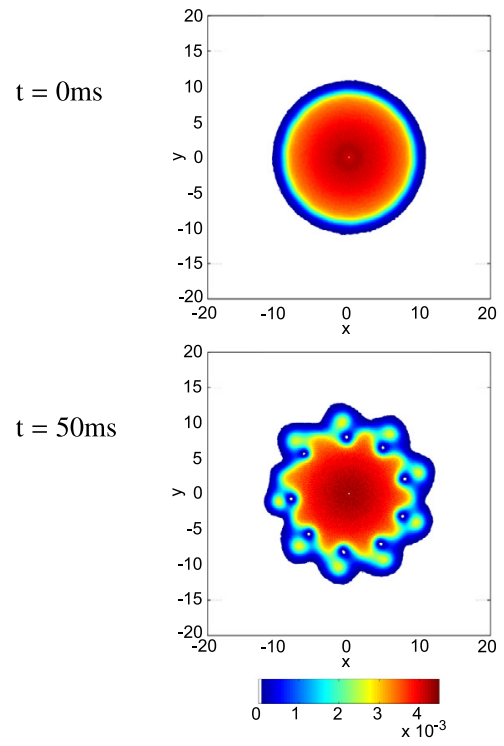

Density of component 1
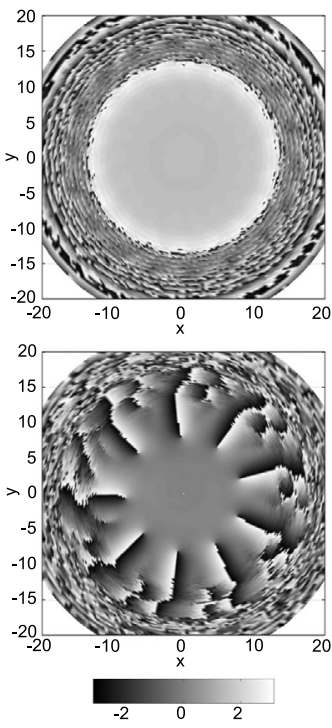

Phase of component 1
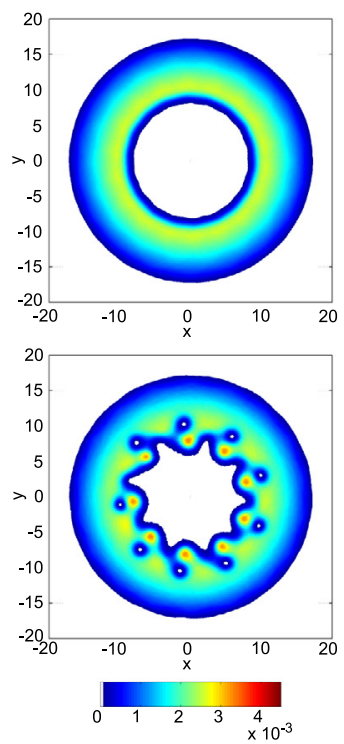

Density of component 2
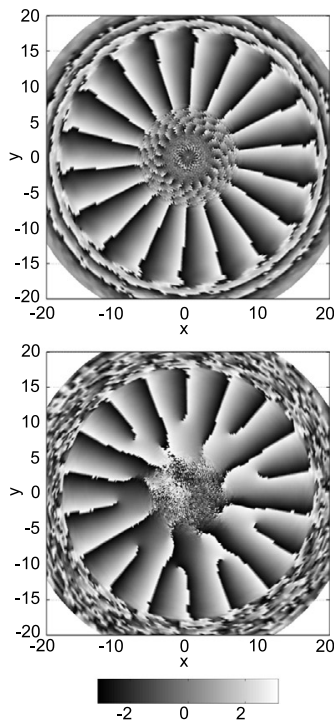

Phase of component 2

Fig. 11. Time evolution of density and phase contours for two-component condensate with $\Omega_{z}=0, \lambda_{\text {tr }}=0$. The initial phase imprinted on the second component causes the second component to rotate relative to the first component and gives rise to the onset of a Kelvin-Helmholtz instability.

To further illustrate the capability of our numerical scheme, we have also performed a time-dependent simulation of the onset of the Kelvin-Helmholtz instability in a trapped 2D two-component condensate as considered by [48]. This test case provides an ideal benchmark problem permitting both a qualitative and quantitative evaluation of the correct implementation of the scheme. We will use similar parameters to the study of Suzuki et al. in which an atomic condensate consisting of a total of $N=1.63 \times 10^{6}{ }^{23} \mathrm{Na}$ atoms was modelled. We assumed the gas is made up of atoms in two different hyperfine states that make up the two components with an equal population in each component. The gas is taken to be trapped in an axisymmetric harmonic potential where the radial and axial trap frequencies are $\omega_{\perp}=2 \pi \times 80 \mathrm{~Hz}$ and $\omega_{z}=2 \pi \times 4 \mathrm{kHz}$, respectively. The strong trapping in the $z$ direction effectively freezes out the dynamics in this direction allowing us to consider effectively two-dimensional dynamics in our simulations. Following [48], we have set the scattering lengths to $a_{11} \simeq 53.4 a_{B}, a_{12}=a_{22} \simeq 54.2 a_{B}$ with $a_{B}$ being the Bohr radius. These parameters favour a phase-separated two-component condensate. Using these parameters, we prepare the initial state of the system by first integrating the equations forward in imaginary time subject to the constraint on the number of particles using the same approach to that described above. Since our parameters lead to phase separation of the two components and $a_{22}>a_{11}$, we find that the ground state consists of component 1 occupying the centre of the trap and is surrounded by component 2 in the form of a ring-shaped condensate as shown in Fig. 11 for $t=0$. After computing the ground states, we imprint a phase on the wavefunction of the second component so that $\psi_{2}=\left|\psi_{2, \mathrm{gs}}\right| \mathrm{e}^{i n_{2} \theta}$ where $\psi_{2, \mathrm{gs}}$ denotes the ground state wavefunction for the second component. We set $n_{2}=18$ which produces an overall rotation in the second component. Our initial condition, that we use for the forward time integration, now consists of two phase-separated components with a circular interface and with the outer component rotating relative to the inner component. This provides the key ingredients necessary for the onset of the Kelvin-Helmholtz instability. To break the axisymmetry, we add small numerical noise to our wavefunctions in the form of $\psi_{\text {noise }}(r, \theta)=0.001 \mathrm{e}^{i 2 \pi R n(r, \theta)}$ where $R n(r, \theta)$ is a randomly sampled function with a uniform distribution. These new wavefunctions are used as the initial conditions for the density and the phase and is shown in Fig. 11 for $t=0$. The initial condition is integrated forward in time using our numerical scheme for the 2D two-component condensate with a time step of $\Delta t=0.0001$. We observe that after some time, a Kelvin-Helmholtz instability develops at the interface between the two components. As time progresses, the instability grows leading to the formation of well defined vortices at the interface. Our results are in perfect qualitative agreement with those presented by Suzuki et al. As a further quantitative check of our results, we note that nine vortices are produced at the interface in our simulations which are in direct agreement with the most unstable azimuthal mode as predicted from a linear stability analysis in [48]. We therefore conclude that our method reproduces the correct temporal behaviour for this case.

As an illustrative example of our 3D scheme, we will present time-dependent simulations of a 3D condensate. We begin by repeating the precessing vortex problem in 3D using $\gamma^{(3 \mathrm{D})}=1.2 \times 10^{4}$ and a grid of $96 \times 64 \times 32$ points in the $(r, \theta, \phi)$ directions respectively. We begin by imprinting a single quantised vortex aligned along the $z$ direction at $r=2$ and $\theta=0$. This initial condition is then integrated forward in time using a time step of $\Delta t=0.005$. As for the $2 \mathrm{D}$ case, the vortex precesses around the centre of the trap as indicated by the final vortex position shown in Fig. 12(a). The 3D isometric view of the vortex shown in the figure clearly depicts the nature of these topological defects within the condensate. As for the $2 \mathrm{D}$ 


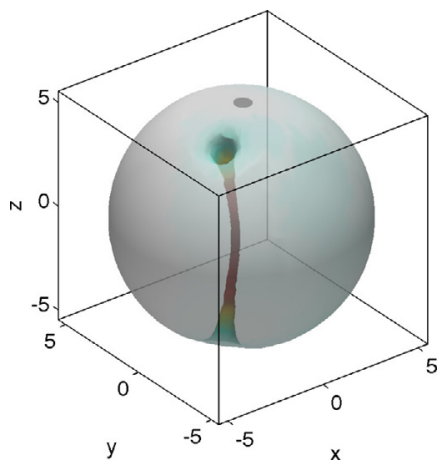

(a) Isosurface at $t=24$

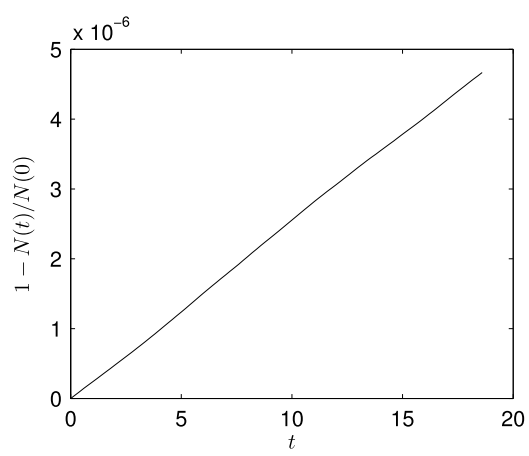

(b) Normalized L2 norm.

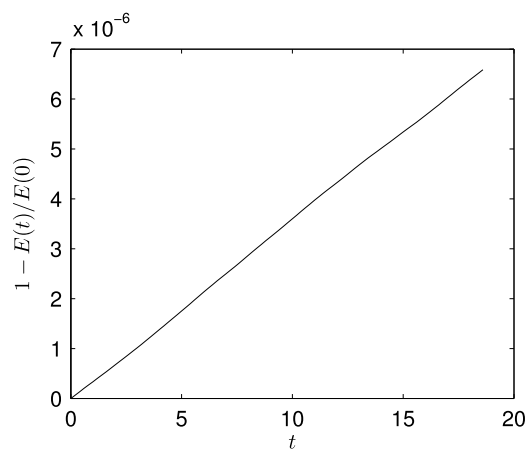

(c) Normalized energy.

Fig. 12. Precessing vortex in a 3D condensate consisting of $20,000{ }^{87} \mathrm{Rb}$ atoms in an isotropic trap with $\omega_{\text {ho }}=50 \mathrm{~Hz}$, and $\lambda_{\text {tr }}=0, \Omega_{z}=0$ : (a) isosurface corresponding to $|\psi|=0.025$ showing nature of single quantised vortex as a topological defect precessing within the condensate for $\left\{\gamma^{(3 \mathrm{D})}=12,000\right.$, $\left.\lambda_{\mathrm{tr}}=0, \Omega_{z}=0\right\}$. The isosurface contouring is given by the function $\sqrt{r}$ where $r$ is the radius measured from the centre of the condensate and therefore reflects the distance that different isosurfaces are located from the centre of the trap; (b) Variation of normalised L2 norm of condensate wavefunction with time; (b) Variation of normalised energy with time.

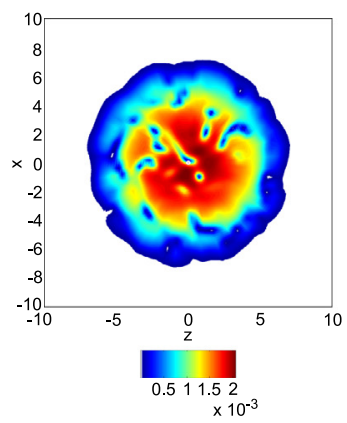

(a) $\mathrm{t}=0$.

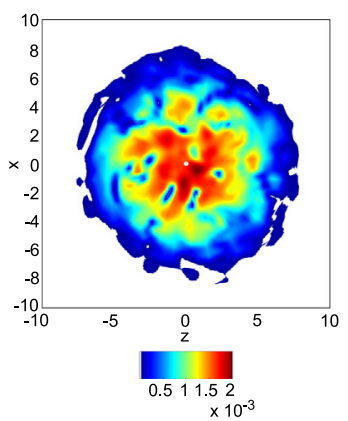

(b) $\mathrm{t}=1.6$

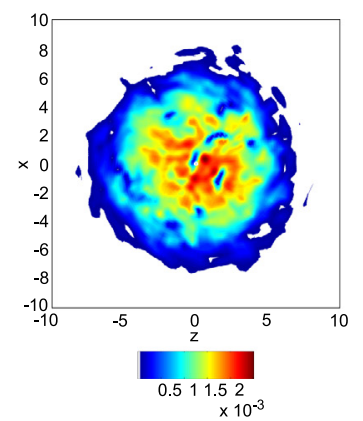

(c) $\mathrm{t}=3.2$

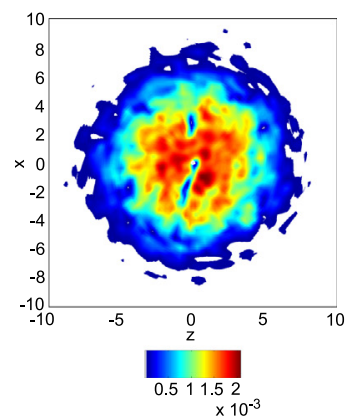

(d) $\mathrm{t}=4.8$

Fig. 13. Cross-sectional density profiles of turbulent condensate state for case with $\left\{\gamma^{(3 \mathrm{D})}=12,000, \lambda_{\mathrm{tr}}=0, \Omega_{z}=0\right\}$.

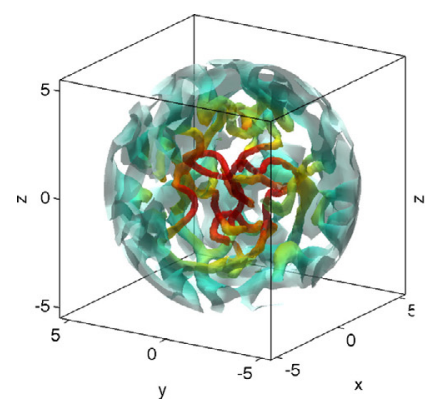

(a) $\mathrm{t}=0$.

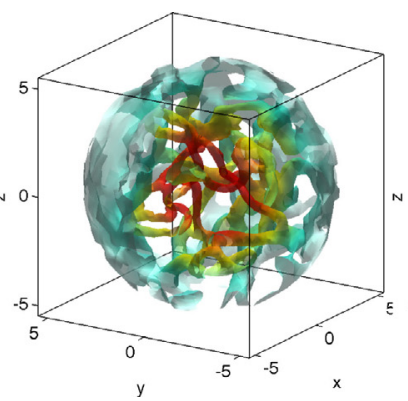

(b) $\mathrm{t}=1.6$

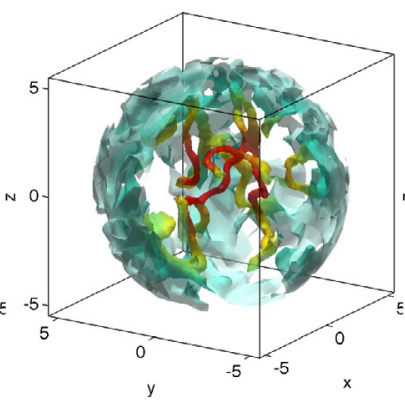

(c) $\mathrm{t}=3.2$

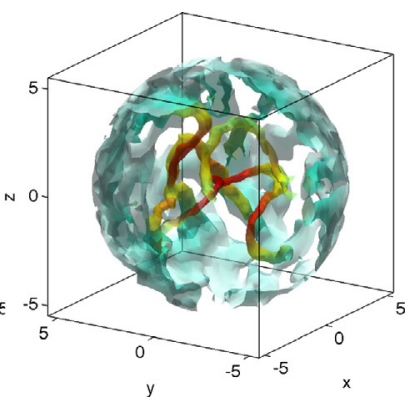

(d) $\mathrm{t}=4.8$

Fig. 14. Condensate isosurface corresponding to $|\psi|=0.025$ showing relaxation of turbulent vortex tangle with time for $\left\{\gamma^{(3 \mathrm{D})}=12,000, \lambda_{\text {tr }}=0, \Omega_{z}=0\right\}$. The isosurface contouring is given by the function $\sqrt{r}$ where $r$ is the radius measured from the centre of the condensate and therefore reflects the distance that different isosurfaces are located from the centre of the trap.

case, we tracked the total number of particles and Hamiltonian with time as shown in Fig. 12(b), (c). As before, we observe that both quantities are conserved to a high accuracy by our numerical method.

We end by simulating the evolution of a vortex tangle in an atomic Bose-Einstein condensate. We prescribe our initial condition by setting $\psi=\psi_{g s} \mathrm{e}^{i 2 \pi R n(r, \theta, \phi)}$ where, as before, $R n(r, \theta, \phi)$ is a randomly sampled function with a uniform distribution. We first relaxed this initial condition through an imaginary time evolution over an interval of $t=0.1$ units using a time step $\Delta t=0.00002$. This has the effect of producing a system at intermediate energies consisting of quantised vortex excitations superimposed on top of a condensate. Cross-sections of the resulting condensate density are shown in Fig. 13. The corresponding 3D view depicting the vortex tangle that is indicative of a condensate in a turbulent state is shown in Fig. 14. Using the initial condition shown in Fig. 13(a) and Fig. 14(a), we evolved the wavefunction under the conservative 
GP equation. The resulting evolution is shown at different times in the figure and illustrates how the tangle relaxes with time. This scenario in which vortices evolve and reconnect and ultimately decay is reminiscent of similar simulations carried out by Berloff and Svistunov [13] but for a homogeneous system.

\section{Conclusions}

We have presented a fully realisable implementation of a numerical scheme based on generalised-Laguerre polynomials with Fourier or spherical harmonics for the solution of the GP multi-component system of equations confined within a harmonic and toroidal trapping potential for 2D and 3D condensates, respectively. A key issue that arises when using generalised-Laguerre polynomials is how to truncate the modes corresponding to different angular wavenumbers. We have shown that a truncation based on the energy spectrum of the linear operator leads to a self-consistent and accurate numerical scheme. An efficient implementation was accomplished by reducing the pseudospectral scheme to matrix-vector operations that could easily be implemented using MATLAB. An important feature of the discretisation presented is the improved accuracy one achieves when combined with an operator-splitting method for the time integration of the governing equation in comparison to methods that have previously been presented in the literature.

Using recently derived theoretical results for the ground state properties of a Bose gas in a harmonic trap with and without rotation, we have been able to demonstrate excellent agreement with our numerical results obtained using our scheme. Furthermore, in contrast to previous work, our approach circumvents the difficulties that arise from having different Laguerre-Lobotto quadrature points for generalised-Laguerre polynomials that correspond to different angular/centrifugal number. This allows us to apply our method to fully 2D and 3D numerical simulations resulting in a fully general and accurate scheme by solving the GP equation in the most natural coordinate system for describing the geometry of BECs that are typically found in a number of experiments. We have demonstrated the full applicability of the method by studying the time-dependent dynamics of vortex lattice formation in 2D, and the motion of a quantised vortex in a spherical BEC.

Given that the scheme we have presented uses a single-particle basis allowing direct control over the cut-off of the energy spectrum, the method will be ideal for use with finite temperature models of BECs. In modelling finite temperature effects, this level of numerical control is essential in order to recover the correct equilibrium properties of the system $[44,16]$. The scheme we have presented is, therefore, likely to find applications in these other areas of atomic physics where thermodynamics and nonequilibrium condensate growth form an important area of research. In addition, the modelling of vortex dynamics that we have focused on in this work is known to be of essence to an understanding of the problem of superfluid turbulence and provides another promising area of application of the numerical scheme presented here.

\section{Appendix A. Non-dimensionalisation of the governing equations of motion}

We are interested in modelling the dynamics of a weakly interacting one- or two-component Bose-condensed gas at zero temperature $(T=0)$. In the presence of a confining external potential, the dynamics of the condensate wavefunctions $\psi_{\alpha}(\mathbf{x}, t)$, where $\alpha=1,2$ for the two different components, can be described by two coupled Gross-Pitaevskii (GP) [40] equations which are given by

$$
\begin{aligned}
i \hbar \frac{\partial \psi_{1}(\mathbf{x}, t)}{\partial t}= & \frac{-\hbar^{2}}{2 m_{1}} \nabla^{2} \psi_{1}(\mathbf{x}, t)+\tilde{V}_{1}(\mathbf{x}) \psi_{1}(\mathbf{x}, t)+U_{11}^{(d)}\left|\psi_{1}(\mathbf{x}, t)\right|^{2} \psi_{1}(\mathbf{x}, t) \\
& +U_{12}^{(d)}\left|\psi_{2}(\mathbf{x}, t)\right|^{2} \psi_{1}(\mathbf{x}, t)-i \hbar \tilde{\boldsymbol{\Omega}} \cdot \mathbf{x} \times \nabla \psi_{1}(\mathbf{x}, t), \\
i \hbar \frac{\partial \psi_{2}(\mathbf{x}, t)}{\partial t}= & \frac{-\hbar^{2}}{2 m_{2}} \nabla^{2} \psi_{2}(\mathbf{x}, t)+\tilde{V}_{2}(\mathbf{x}) \psi_{2}(\mathbf{x}, t)+U_{22}^{(d)}\left|\psi_{2}(\mathbf{x}, t)\right|^{2} \psi_{2}(\mathbf{x}, t) \\
& +U_{21}^{(d)}\left|\psi_{1}(\mathbf{x}, t)\right|^{2} \psi_{2}(\mathbf{x}, t)-i \hbar \tilde{\boldsymbol{\Omega}} \cdot \mathbf{x} \times \nabla \psi_{2}(\mathbf{x}, t),
\end{aligned}
$$

where $d$ denotes the number of spatial dimensions, and $\mathbf{x} \in \mathbb{R}^{d}$ with $\mathbf{x}=(x, y)$ in $2 \mathrm{D}$ and $\mathbf{x}=(x, y, z)$ in $3 \mathrm{D}$, respectively. In addition, $\hbar$ is the reduced Planck's constant, $\tilde{V}_{1}(\mathbf{x})$ and $\tilde{V}_{2}(\mathbf{x})$ are the external trapping potentials, $U_{\alpha \beta}^{(d)}$ are the interatomic and intra-atomic interaction coefficients, and $\tilde{\boldsymbol{\Omega}}$ is the angular rotation vector. We note that the interatomic interactions must satisfy the condition $U_{12}^{(d)}=U_{21}^{(d)}$. For components corresponding to atoms in two different hyperfine states, $m_{1}=m_{2}$. However, for different atomic species, $m_{\alpha}$ would correspond to the atomic mass of each species. In this work, we will exclusively consider the case of a harmonic trapping potential with a possibility of a Gaussian central potential in which case

$$
\tilde{V}_{\alpha}(\mathbf{x})= \begin{cases}\frac{1}{2} m_{\alpha}\left(\omega_{x, \alpha}^{2} x^{2}+\omega_{y, \alpha}^{2} y^{2}\right)+m_{\alpha} \omega_{\mathrm{tr}, \alpha}^{2} \exp \left(\frac{-2\left(x^{2}+y^{2}\right)}{L_{\mathrm{tr}, \alpha}^{2}}\right) & \text { in 2D, } \\ \frac{1}{2} m_{\alpha}\left(\omega_{x, \alpha}^{2} x^{2}+\omega_{y, \alpha}^{2} y^{2}+\omega_{z, \alpha}^{2} z^{2}\right)+m_{\alpha} \omega_{\mathrm{tr}, \alpha}^{2} \exp \left(\frac{-2\left(x^{2}+y^{2}\right)}{L_{\mathrm{tr}, \alpha}^{2}}\right) & \text { in 3D, }\end{cases}
$$

with $\omega_{x, \alpha}, \omega_{y, \alpha}, \omega_{z, \alpha}$ being the harmonic trap frequencies for the two components in the $x, y$, and $z$ directions respectively. The strength of the Gaussian potential located at the centre is determined by the toroidal trapping frequency $\omega_{\operatorname{tr}, \alpha}$, and the width is characterised by $L_{\mathrm{tr}, \alpha}$. The above equation is then supplemented by the boundary conditions 


$$
\psi_{\alpha} \rightarrow 0 \text { as }|\mathbf{x}| \rightarrow \infty,
$$

which is a consequence of the harmonic trapping potentials $\tilde{V}_{\alpha}(\mathbf{x}) \rightarrow \infty$ as $|\mathbf{x}| \rightarrow \pm \infty$.

The interaction parameters $U_{\alpha \beta}^{(d)}$ are defined differently in 2D and 3D. In 3D, $U_{\alpha \beta}^{(3)}=2 \pi \hbar^{2} a_{s, \alpha \beta}\left(m_{\alpha}+m_{\beta}\right) / m_{\alpha} m_{\beta}$ and are related to the $s$-wave scattering lengths $a_{s, \alpha \beta}$ which can typically be varied in experiments through the use of Feshbach resonance [40]. The interaction parameters in $2 \mathrm{D}$ are then given by $U_{\alpha \beta}^{(2)}=U_{\alpha \beta}^{(3)}\left(m_{\alpha} \omega_{z, \alpha} /(2 \pi \hbar)\right)^{1 / 2}$. The GP system of equations has a number of important conserved quantities including the total number of particles in each component and the total energy (Hamiltonian) which are given respectively by [25]

$$
\begin{aligned}
N_{\alpha}= & \int_{\mathbb{R}^{d}}\left|\psi_{\alpha}(\mathbf{x}, t)\right|^{2} d^{d} \mathbf{x}, \\
H= & \int_{\mathbb{R}^{d}}\left[\sum_{\alpha=1}^{2}\left(\frac{\hbar^{2}}{2 m_{\alpha}}\left|\nabla \psi_{\alpha}(\mathbf{x}, t)\right|^{2}+\tilde{V}_{\alpha}(\mathbf{x})\left|\psi_{\alpha}(\mathbf{x}, t)\right|^{2}+\frac{U_{\alpha, \alpha}^{(d)}}{2}\left|\psi_{\alpha}(\mathbf{x}, t)\right|^{4}\right)+U_{12}^{(d)}\left|\psi_{1}(\mathbf{x}, t)\right|^{2}\left|\psi_{2}(\mathbf{x}, t)\right|^{2}\right] d^{d} \mathbf{x} \\
& -i \hbar \int_{\mathbb{R}^{d}}\left[\sum_{\alpha=1}^{2} \psi_{\alpha}^{*} \tilde{\boldsymbol{\Omega}} \cdot \mathbf{x} \times \nabla \psi_{\alpha}(\mathbf{x}, t)\right] d^{d} \mathbf{x} .
\end{aligned}
$$

We will non-dimensionalise the above equations using the oscillator length scale $\left(a_{h o}=\sqrt{\hbar /\left(m_{1} \omega_{h o}\right)}\right)$, and the oscillator frequency given by $\omega_{h o}=\left(\omega_{x, 1} \omega_{y, 1} \omega_{z, 1}\right)^{1 / 3}$ in $3 \mathrm{D}$, and $\omega_{h o}=\left(\omega_{x, 1} \omega_{y, 1}\right)^{1 / 2}$ in $2 \mathrm{D}$. Then rescaling space and time such that

$$
t \rightarrow t / \omega_{h o}, \quad \mathbf{x} \rightarrow \mathbf{x} a_{h o},
$$

and normalising the wavefunctions to unity by setting $\psi_{\alpha} \rightarrow \sqrt{N}_{\alpha} \psi_{\alpha}$, we obtain

$$
\begin{aligned}
& i \frac{\partial \psi_{1}(\mathbf{x}, t)}{\partial t}=\left[\frac{-1}{2} \nabla^{2}+V_{1}(\mathbf{x})+\gamma_{11}^{(d)}\left|\psi_{1}(\mathbf{x}, t)\right|^{2}+\gamma_{12}^{(d)}\left|\psi_{2}(\mathbf{x}, t)\right|^{2}-i \mathbf{x} \cdot \boldsymbol{\Omega} \times \nabla\right] \psi_{1}(\mathbf{x}, t), \\
& i \frac{\partial \psi_{2}(\mathbf{x}, t)}{\partial t}=\left[\frac{-\delta}{2} \nabla^{2}+\frac{1}{\delta} V_{2}(\mathbf{x})+\gamma_{22}^{(d)}\left|\psi_{2}(\mathbf{x}, t)\right|^{2}+\gamma_{21}^{(d)}\left|\psi_{1}(\mathbf{x}, t)\right|^{2}-i \mathbf{x} \cdot \boldsymbol{\Omega} \times \nabla\right] \psi_{2}(\mathbf{x}, t),
\end{aligned}
$$

with

$$
V_{\alpha}(\mathbf{x})= \begin{cases}\frac{1}{2}\left(\lambda_{x, \alpha}^{2} x^{2}+\lambda_{y, \alpha}^{2} y^{2}\right)+\lambda_{\operatorname{tr}, \alpha} \exp \left(\frac{-2\left(x^{2}+y^{2}\right)}{l_{\mathrm{tr}, \alpha}^{2}}\right) & \text { in 2D } \\ \frac{1}{2}\left(\lambda_{x, \alpha}^{2} x^{2}+\lambda_{y, \alpha}^{2} y^{2}+\lambda_{z, \alpha}^{2} z^{2}\right)+\lambda_{\operatorname{tr}, \alpha} \exp \left(\frac{-2\left(x^{2}+y^{2}\right)}{l_{\mathrm{tr}, \alpha}^{2}}\right) & \text { in 3D. }\end{cases}
$$

In Eq. (A.7), $\gamma_{\alpha \beta}^{(3)}=2 \pi N_{\beta} a_{s, \alpha \beta}\left(1+m_{\alpha} / m_{\beta}\right) \delta_{\alpha} / a_{h o}$ in 3D whereas $\gamma_{\alpha \beta}^{(2)}=\gamma_{\alpha \beta}^{(3)} a_{h o}\left(m_{\alpha} \omega_{z, \alpha} / 2 \pi \hbar\right)^{1 / 2}$ in $2 \mathrm{D}, \lambda_{x, \alpha}=\omega_{x, \alpha} / \omega_{h o}$, $\lambda_{y, \alpha}=\omega_{y, \alpha} / \omega_{h o}, \lambda_{z, \alpha}=\omega_{z, \alpha} / \omega_{h o}, \lambda_{\operatorname{tr}, \alpha}=\omega_{\operatorname{tr}, \alpha} / \omega_{h o}, \boldsymbol{\Omega}=\tilde{\boldsymbol{\Omega}} / \omega_{h o}, l_{\mathrm{tr}, \alpha}=L_{\mathrm{tr}, \alpha} / a_{h o}, \delta=m_{1} / m_{2}>1$ (i.e. we set component one to correspond to the gas with the larger of the two masses). For later convenience, we have introduced the parameter $\delta_{\alpha}$ such that $\delta_{1}=1$ and $\delta_{2}=\delta$. These equations can be solved subject to the initial conditions $\psi_{\alpha}(\mathbf{x}, 0)=\psi_{0, \alpha}(\mathbf{x})$.

In the limit where the harmonic trap oscillator frequency in one direction becomes significantly strong, the dynamics of the condensate will be severely restricted to occur essentially on the plane spanned by the vectors normal to that direction. Without loss of generality, we can take this direction to be along the $z$-coordinate axis, such that $\omega_{z} \gg \omega_{x}$, $\omega_{y}$. In this case the dynamics reduces to motion on the $x-y$ plane. Moreover, since we will be interested in simulating rotating condensates we can take without loss of generality $\boldsymbol{\Omega}=\left(0,0, \Omega_{z}\right)$. We, will therefore consider cases where the respective equations will be solved in $2 \mathrm{D}$ as well as $3 \mathrm{D}$. In the former, we will work with a polar $(r, \theta)$ coordinate system whereas in the latter, we will revert to a spherical coordinate system denoted by $(r, \theta, \phi)$. After re-expressing our wavefunctions in terms of polar $(\psi(\mathbf{x}, t) \rightarrow \psi(\mathbf{r}, t) \equiv \psi(r, \theta, t))$, and spherical $(\psi(\mathbf{x}, t) \rightarrow \psi(\mathbf{r}, t) \equiv \psi(r, \theta, \phi, t))$ coordinates, we recover Eqs. (2) and (5), respectively.

\section{References}

[1] M. Abramowitz, I.A. Stegun, Handbook of Mathematical Functions with Formulas, Graphs, and Mathematical Tables, Dover Publications, New York, 1972.

[2] A. Aftalion, P. Mason, Rotation of a Bose-Einstein condensate held under a toroidal trap, Phys. Rev. A 81 (2010) 023607.

[3] M.H. Anderson, J.R. Ensher, M.R. Matthews, C.E. Wieman, E.A. Cornell, Observation of Bose-Einstein condensation in a dilute atomic vapor, Science 269 (5221) (1995) 198-201.

[4] G.E. Astrakharchik, L.P. Pitaevskii, Motion of a heavy impurity through a Bose-Einstein condensate, Phys. Rev. A 70 (2004) 013608.

[5] W. Bao, D. Jaksch, An explicit unconditionally stable numerical method for solving damped nonlinear Schrödinger equations with a focusing nonlinearity, SIAM J. Numer. Anal. 41 (2003) 1406-1426. 
[6] W. Bao, J. Shen, A fourth-order time-splitting Laguerre-Hermite pseudospectral method for Bose-Einstein condensates, SIAM J. Sci. Comput. 26 (6) (2005) 2010-2028.

[7] W. Bao, J. Shen, A generalized-Laguerre-Hermite pseudospectral method for computing symmetric and central vortex states in Bose-Einstein condensates, J. Comput. Phys. 227 (2008) 9778-9793.

[8] W. Bao, H. Li, J. Shen, A generalized-Laguerre-Fourier-Hermite pseudospectral method for computing the dynamics of rotating Bose-Einstein condensates, SIAM J. Sci. Comput. 31 (2009) 3685-3711.

[9] W. Bao, Y. Cai, Mathematical theory and numerical methods for Bose-Einstein condensates, Kinet. Relat. Models 6 (2013) 1-135.

[10] R. Barnett, G. Refael, M. Porter, H.P. Büchler, Vortex lattice locking in rotating two-component Bose-Einstein condensates, New J. Phys. 10 (2008) 043030.

[11] R. Barnett, E. Chen, G. Refael, Vortex synchronization in Bose-Einstein condensates: A time-dependent Gross-Pitaevskii equation approach, New J. Phys. 12 (2010) 043004.

[12] D. Baye, P.-H. Heenen, Generalised meshes for quantum mechanical problems, J. Phys. A, Math. Gen. 19 (1986) 2041-2059.

[13] N.G. Berloff, B.V. Svistunov, Scenario of strongly non-equilibrated Bose-Einstein condensation, Phys. Rev. A 66 (2002) 013603.

[14] N.G. Berloff, Padé approximations of solitary wave solutions of the Gross-Pitaevskii equation, J. Phys. A, Math. Gen. 37 (2004) $1617-1632$.

[15] P.B. Blakie, Numerical method for solving the projected Gross-Pitaevskii equation, Phys. Rev. E 78 (2008) 026704.

[16] P.B. Blakie, A.S. Bradley, M.J. Davis, Dynamics and statistical mechanics of ultra-cold Bose gases using c-field techniques, Adv. Phys. 57 (2008) $363-455$.

[17] A.G. Borisov, Solution of the radial Schrödinger equation in cylindrical and spherical coordinates by mapped Fourier transform algorithms, J. Chem. Phys. 114 (18) (2001) 7770-7777.

[18] J.P. Boyd, C. Rangan, P.H. Bucksbaum, Pseudospectral method on a semi-infinite interval with application to a hydrogen atom: A comparison of the mapped Fourier-sine method with Laguerre series and rational Chebyshev expansions, J. Comput. Phys. 188 (2003) 56-74.

[19] C.C. Bradley, C.A. Sackett, J.J. Tollett, R.G. Hulet, Evidence of Bose-Einstein condensation in an atomic gas with attractive interactions, Phys. Rev. Lett. 75 (9) (1995) 1687-1690.

[20] A.S. Bradley, C.W. Gardiner, M.J. Davis, Bose-Einstein condensation from a rotating thermal cloud: Vortex nucleation and lattice formation, Phys. Rev. A 77 (2008) 033616.

[21] P.J. Channell, C. Scovel, Symplectic integration of Hamiltonian systems, Nonlinearity 3 (1990) 231-259.

[22] G.C. Corey, Pseudospectral method for solving the time-dependent Schrödinger equation in spherical coordinates, J. Chem. Phys. 97 (6) (1992) $4115-4126$.

[23] J.R. Discoll, D. Healy, Computing Fourier transforms and convolutions on the 2-sphere, Adv. Appl. Math. 15 (1994) 202.

[24] M. Edwards, P.A. Ruprecht, K. Burnett, R.J. Dodd, C.W. Clark, Collective excitations of atomic Bose-Einstein condensates, Phys. Rev. Lett. 77 (1996) $1671-1674$.

[25] A.L. Fetter, Rotating trapped Bose-Einstein condensates, Rev. Mod. Phys. 81 (2009) 647.

[26] B. Fornberg, A Practical Guide to Pseudospectral Methods, 1998.

[27] V.M. Pérez-García, X. Liu, Numerical methods for the simulation of trapped nonlinear Schrödinger systems, Appl. Math. Comput. 144 (2003) $215-235$.

[28] D. Gottlieb, A. Orszag, Numerical Analysis of Spectral Methods: Theory and Applications, 1977.

[29] E.P. Gross, Structure of a quantized vortex in boson systems, Nuovo Cimento 20 (3) (May 1961) 454-457.

[30] M.R. Hermann, J.A. Fleck Jr., Split-operator spectral method for solving the time-dependent Schrödinger equation in spherical coordinates, Phys. Rev. A 38 (12) (1988) 6000-6012.

[31] T. Hyouguchi, S. Adachi, M. Ueda, Divergence-free WKB method, Phys. Rev. Lett. 88 (2002) 170404.

[32] S. Jiang, L. Greengard, Efficient representation of nonreflecting boundary conditions for the time-dependent Schrödinger equation in two dimensions, Commun. Pure Appl. Math. 61 (2) (2008) 261-288.

[33] D.S. Jin, J.R. Ensher, M.R. Matthews, C.E. Wieman, E.A. Cornell, Collective excitations of a Bose-Einstein condensate in a dilute gas, Phys. Rev. Lett. 77 (1996) 420-423.

[34] K. Kasamatsu, M. Tsubota, Quantized vortices in atomic Bose-Einstein condensates, in: M. Tsubota (Ed.), Prog. Low Temp. Phys., Quantum Turbul., vol. XVI, Elsevier, 2009.

[35] P.G. Kevrekidis, D.J. Frantzeskakis, R. Carretero-González (Eds.), Emergent Nonlinear Phenomena in Bose-Einstein Condensates: Theory and Experiment, Springer-Verlag, 2008.

[36] R.I. McLachlan, R.W. Quispel, Splitting methods, Acta Numer. 11 (2002) 341-434.

[37] R.I. McLachlan, R.W. Quispel, Geometric integrators for ODEs, J. Phys. A, Math. Gen. 39 (2006) 5251-5285.

[38] G.M. Muslu, H.A. Erbay, Higher-order split-step Fourier schemes for the generalized nonlinear Schrödinger equation, Math. Comput. Simul. 67 (2005) $581-595$.

[39] D. Pathria, J.LL. Morris, Pseudo-spectral solution of nonlinear Schrödinger equations, J. Comput. Phys. 87 (1990) 108-125.

[40] C.J. Pethick, H. Smith, Bose-Einstein Condensation in Dilute Gases, Cambridge University Press, 2008.

[41] L.P. Pitaevskii, Vortex lines in an imperfect Bose gas, Zh. Eksp. Teor. Fiz. 40 (1961) 646-649 (in Russian); Sov. Phys. JETP 13 (1961) $451-454$ (in English).

[42] L.P. Pitaevskii, S. Stringari, Bose-Einstein Condensation, Clarendon, Oxford, 2003.

[43] N.P. Proukakis, B. Jackson, Finite-temperature models of Bose-Einstein condensation, J. Phys. B, At. Mol. Opt. Phys. 41 (2008) 203002.

[44] H. Salman, Approximating steady states in equilibrium and nonequilibrium condensates, Phys. Rev. A 85 (2012) 063622.

[45] J. Shen, Stable and efficient spectral methods in unbounded domains using Laguerre functions, SIAM J. Numer. Anal. 38 (4) (2000) $1113-1133$.

[46] T. Sørevik, T. Birkeland, G. Okša, Numerical solution of the 3D time dependent Schrödinger equation in spherical coordinates: Spectral basis and effects of split-operator technique, J. Comput. Appl. Math. 225 (2009) 56-67.

[47] G. Strang, On the construction and comparison of difference schemes, SIAM J. Numer. Anal. 5 (1968) 506-517.

[48] N. Suzuki, H. Takeuchi, K. Kasamatsu, M. Tsubota, H. Saito, Crossover between Kelvin-Helmholtz and counter-superflow instabilities in two-component Bose-Einstein condensates, Phys. Rev. A 82 (2010) 063604.

[49] T.R. Taha, M.J. Ablowitz, Analytical and numerical aspects of certain nonlinear evolution equations. II. Numerical nonlinear Schrödinger equation, J. Comput. Phys. 55 (1984) 203-230.

[50] J.A.C. Weideman, B.M. Herbst, Split-step methods for the solution of the nonlinear Schrödinger equation, SIAM J. Numer. Anal. 23 (3) (1986) $485-507$. 